\title{
Synthesis of P-Chirogenic Diphosphinotriazoles and Their Use in Asymmetric Catalysis
}

Jérôme Bayardon, ${ }^{*}[a]$ Yoann Rousselin, [a] and Raluca Malacea-Kabbara [a]

[a] Institut de Chimie Moléculaire de I'Université de Bourgogne- Franche-Comté, ICMUB-OCS (UMRCNRS 6302), 19 avenue A. Savary BP 47870, 21078 Dijon CEDEX, France

E-mail: jerome.bayardon@u-bourgogne.fr

Supporting information for this article is given via a link at the end of the document.

Abstract: The stereoselective synthesis of P-chirogenic diphosphinotriazoles using ephedrine methodology was described. The coordination behavior of these compounds as $P, P$-ligands has been demonstrated by the preparation as well as the spectroscopic and X-ray crystallographic analyses of a palladium complex. The efficiency of these new P-chirogenic diphosphines in the palladiumcatalyzed asymmetric allylic substitution reaction was also evaluated.

\section{Introduction}

In the last few decades, 1,2,3-triazole-based diphosphines, in which the phosphorus moieties were incorporated on triazole ring and/or onto exocyclic groups, have been studied, especially in organometallic chemistry and in catalysis. ${ }^{[1]}$ For example (Figure 1), Gandelman's group described in 2008 the synthesis of bis-phosphinotriazole 1 for its application as PCP pincer complexes in Heck reaction. ${ }^{[2]}$ Later on, the same group reported the synthesis of the diphosphinotriazole 2 and its corresponding platinum complex. ${ }^{[3]}$ In 2015, Manoury and Virieux synthesized diphosphine supported by bis(triazole)backbone $\mathbf{3}$ in order to studied its coordination chemistry toward transition metals ${ }^{[4]}$. Recently, Balakrishna and co-workers synthesized 1,2,3-triazole-based diphosphine 4 for its use in Pd-catalyzed C-C coupling reactions. ${ }^{[5]}$ Finally, the chiral ferrocenyl triazole-based diphosphines 5, named ClickFerroPhos, were used as $P, P$-bidentate ligands in rhodium- and ruthenium-catalyzed asymmetric hydrogenation of alkenes and ketones as well as in asymmetric 1,3dipolar cycloaddition of azomethine ylide catalysed by copper complex. ${ }^{[6]}$ These chiral diphosphines, possessing central and planar chirality, are, to the best of our knowledge, the only examples of chiral diphosphinotriazoles reported so far. ${ }^{[7]}$ Consequently, none of them are P-chirogenic. ${ }^{[8]} \mathrm{P}$-chirogenic diphosphines are quite interesting compounds which have been extensively employed especially in asymmetric catalysis as chiral ligands. ${ }^{[9]}$ For example, the use of DiPAMP, ${ }^{[10]}$ QuinoxP* ${ }^{[11]}$ MiniPhos $^{[12]}$ and BIBOP ${ }^{[13]}$ and their derivatives ${ }^{[9]]}$ has led to excellent enantioselectivities in various asymmetric transformations (Figure 2).

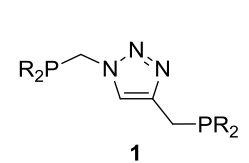

(Gandelman)<smiles></smiles>

2

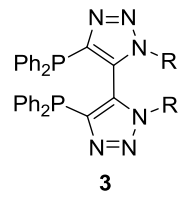

(Manoury and Virieux)

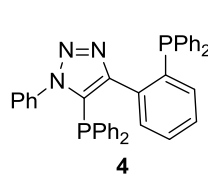

(Balakrishna)

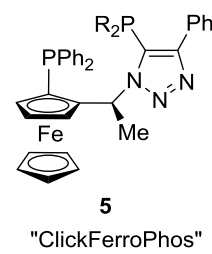

Figure 1. Examples of 1,2,3-trizole-based diphosphines.<smiles>[O+]P(CCP([Pb]O)c1ccccc1)c1ccccc1</smiles>

DiPAMP<smiles>CP(Pc1nc2ccccc2nc1P(C)C(C)(C)C)C(C)(C)C</smiles>

QuinoxP*<smiles>CP(CP[Pb](C)(C)C)C(C)(C)C</smiles>

MiniPhos

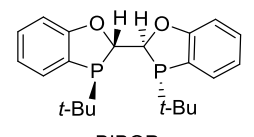

BIBOP 
We have described in our group, the synthesis of $\mathrm{P}$-chirogenic diphosphines ${ }^{[14]}$ and more recently an 1,2,3-triazole based-monophosphine ${ }^{[8 c]}$ for their application as ligands in asymmetric catalysis. In continuation of this work on Pchirogenic ligands, we will report herein the preparation of P-chirogenic diphosphinotriazoles in which the two phosphorus centres were linked to the triazole backbone (Figure 3) and their use as chiral ligands in Pd-catalyzed asymmetric allylic substitution reactions.

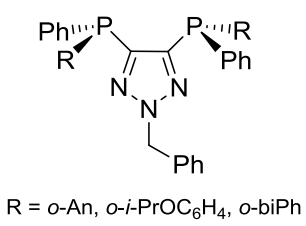

6

Figure 3. Structure of P-chirogenic diphosphinotriazoles 6

\section{Results and Discussion}

The new P-chirogenic 1,2,3-triazole-based diphosphines 6a-c have been synthesized according to the well-established ephedrine methodology starting from $(2 R p, 4 S, 5 R)-(+)-3,4$-dimethyl-2,5-diphenyl-1,3,2-oxazaphospholidine $7^{[14 a],[15]}$ as initial auxiliary to induce chirality at phosphorus centres (Scheme 1$)$.

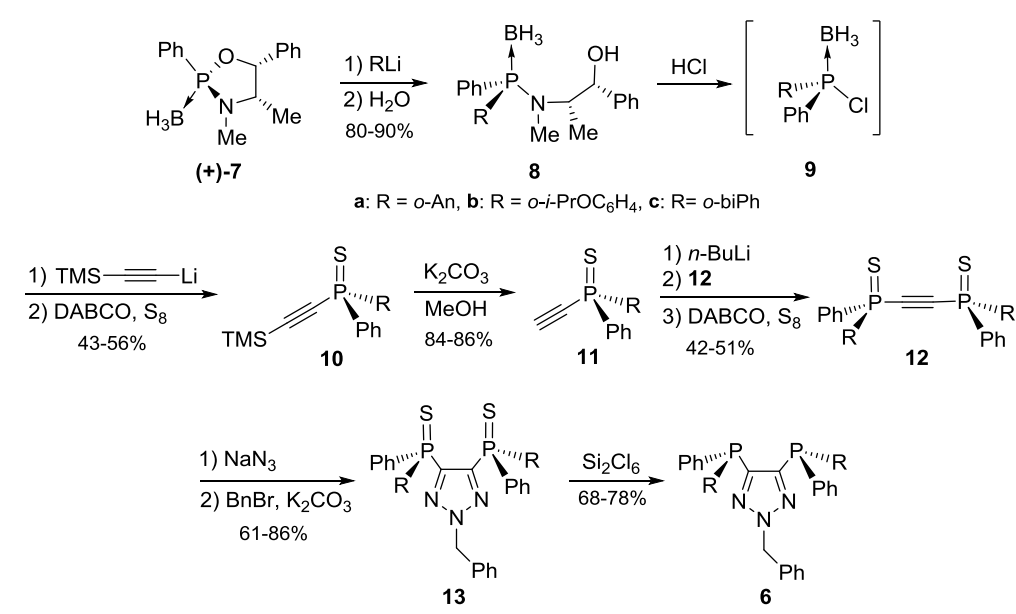

Scheme 1. Synthetic route to P-chirogenic diphosphinotriazoles 6a-c.

The reaction of the complex (+)-7 with organolithium reagents RLi stereospecifically leads, after P-O bond cleavage, to the corresponding aminophosphine-boranes $8 \mathrm{a}-\mathbf{c}$ in $80-90 \%$ yields. The chlorophosphine-boranes 9a-c, which are readily obtained by acidolysis of the aminophosphine-boranes 8a-c with dry $\mathrm{HCl}^{[16]}$ reacted successively with 2(trimethylsilyl)ethynyllithium ${ }^{[17]}$ then with DABCO in presence of elementary sulfur at $50^{\circ} \mathrm{C}$ to provide $\mathrm{P}$-chirogenic phosphine-sulfides $10 a-c$ in $43-56 \%$ overall yields. Their analysis by HPLC on chiral column $(99 \%$ ee for each compound), proves that the reaction sequence proceeds without racemization at the P-centre. The desilylation of phosphine-sulfides 10a-c was readily achieved by treatment with $\mathrm{K}_{2} \mathrm{CO}_{3}$ in $\mathrm{MeOH} / \mathrm{THF}$ mixture at room temperature. The resulting terminal alkynes $11 \mathrm{a}-\mathrm{c}$ were isolated in $84-86 \%$ after purification by chromatography on silica gel. The deprotonation of $11 \mathrm{a}-\mathrm{c}$ in presence of $n$-BuLi at $-78^{\circ} \mathrm{C}$ followed by reaction with chlorophosphine-boranes $9 \mathrm{a}-\mathrm{c}$ then with DABCO and $\mathrm{S}_{8}$ at $50^{\circ} \mathrm{C}$ afford the $\mathrm{C} 2$-symmetric bis(thiophosphinyl)acetylenes $12 \mathrm{a}-\mathrm{c}$ in $42-51 \%$ overall yields. ${ }^{[18]}\left\{{ }^{1} \mathrm{H}\right\}^{31} \mathrm{P}$ and ${ }^{1} \mathrm{H}$ NMR analyses of compounds 12a-c (see Supporting Information) showed no signal corresponding to meso form, proving that no racemization occurred at the P-centres during the two-steps reaction sequence. Moreover, crystals of 12a suitable for $X$-ray analysis have been obtained and the $X$-ray structure is depicted in figure 4 .

It is important to note that the use of sulfide protecting group on P-centre is of great interest because it allows, in the case P-chirogenic phosphine-sulfides $\mathbf{1 0 a - c}$ and bis(thiophosphinyl)acetylenes 12a-c, to obtain more stable compounds than their borane analogues which are obtained partially decomplexed during the synthesis. ${ }^{[19]}$ Moreover, the presence of sulfide electron-withdrawing group on the both P-centres of compounds $\mathbf{1 2 a - c}$ allows to activate the triple bond during the cycloaddition with sodium azide. ${ }^{[20]}$ 


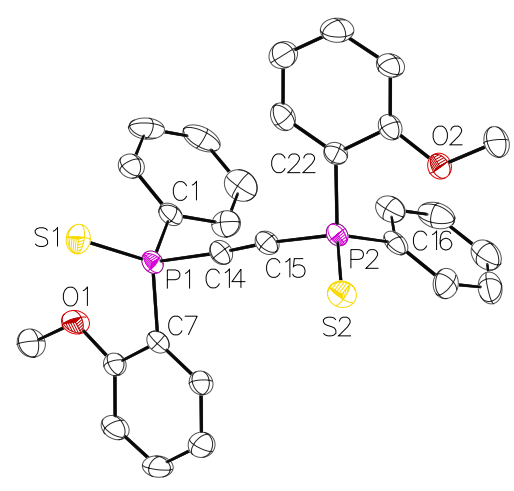

Figure 4. ORTEP view of compound $12 \mathrm{a}[\mathrm{P} 1(R)$ and $\mathrm{P} 2(R)]$. Thermal ellipsoids are drawn at $50 \%$ probability plot. $\mathrm{H}$ atoms are omitted for clarity.

The reaction of P-chirogenic bis(thiophosphinyl)acetylenes $15 \mathrm{a}-\mathrm{c}$ with sodium azide in DMF at $50^{\circ} \mathrm{C}^{[20]}$ then with benzyl bromide in presence of $\mathrm{K}_{2} \mathrm{CO}_{3}$ affords the P-chirogenic 4,5-bis(thiophosphinyl)-1,2,3-triazoles 13a-c in $61-86 \%$ isolated yields. ${ }^{[21]}$ Finally, desulfidation of $13 \mathrm{a}-\mathrm{c}$ was performed by reaction with $\mathrm{Si}_{2} \mathrm{Cl}_{6}{ }^{[22]}$ in toluene at $80^{\circ} \mathrm{C}$ during one hour to give, after recrystallization, P-chirogenic diphosphines $6 \mathrm{a}-\mathrm{c}$ in $68-78 \%$ yields (Scheme 1). The enantiomeric purities of each diphosphinotriazole $6 \mathbf{a}-\mathbf{c}$ were determined by HPLC on chiral column with $99 \%$ ee. Single crystals of $6 \mathbf{a}$ have been obtained by slow diffusion of hexane into dichloromethane and its structure was established by X-ray analysis (Figure 5). The compound 6 a crystallizes in $\mathrm{P} 2{ }_{1}$ chiral space group and the Flack parameter refinement allows the unambiguous determination of the $S$-absolute configuration on the both phosphorus centres. ${ }^{[23]}$

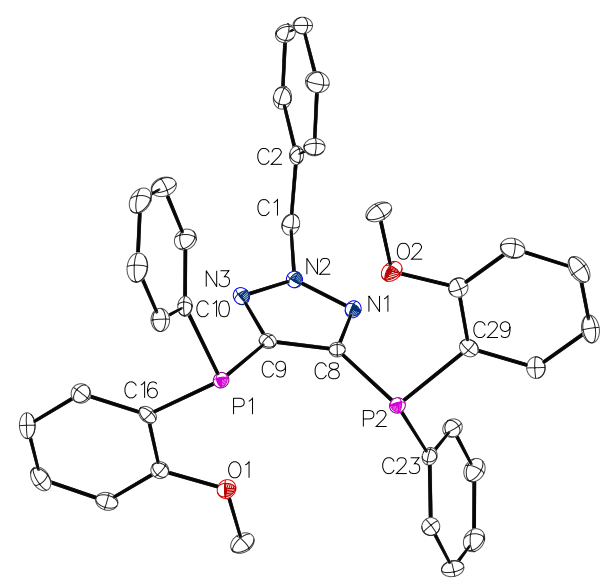

Figure 5. ORTEP view of compound $6 \mathbf{a}[\mathrm{P} 1(S)$ and $\mathrm{P} 2(S)]$. Thermal ellipsoids are drawn at $50 \%$ probability plot. $\mathrm{H}$ atoms are omitted for clarity.

In the next step, we investigated the coordination behavior of P-chirogenic diphosphinotriazoles 6 with late transition metals. Biphosphine 6 a reacted with half equivalent of $[\mathrm{Pd}(\text { allyl }) \mathrm{Cl}]_{2}$ and $\mathrm{AgPF}_{6}$ in a mixture of $\mathrm{CH}_{2} \mathrm{Cl}_{2} / \mathrm{MeOH}$ at room temperature to afford the desired cationic palladium-allylic complex 14 in $78 \%$ yield (Scheme 2).

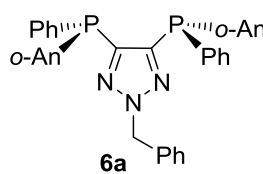<smiles>[PbH2][PbH2]</smiles>
$78 \%$

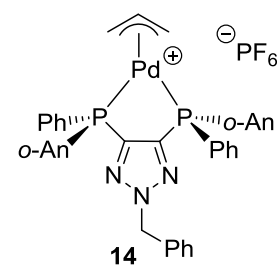


The $\left\{{ }^{1} \mathrm{H}{ }^{31} \mathrm{P}\right.$ NMR spectrum shows a singlet shifted from $-43.7 \mathrm{ppm}$ (for 6a) to $7.2 \mathrm{ppm}$ (for complex 14), clearly indicating coordination of both phosphine arms to the palladium centre. Moreover single crystals of Pd(II) complex 14 suitable for X-ray diffraction analysis were obtained by recrystallization in dichloromethane. The structure of the complex 14 is shown in figure 6. The complex 14, with an unsubstituted allyl ligand, shows the expected square-planar coordination geometry of the $\mathrm{PdC}_{2} \mathrm{P}_{2}$ core and an almost planar conformation of the ligand framework (P2,P3,C27,C28,N1,N2,N3 plane have a RMSD of $0.035 \AA$ ). This structure confirms that ligand 6 a binds palladium through the both phosphorus atoms to form a five-membered ring chelate.

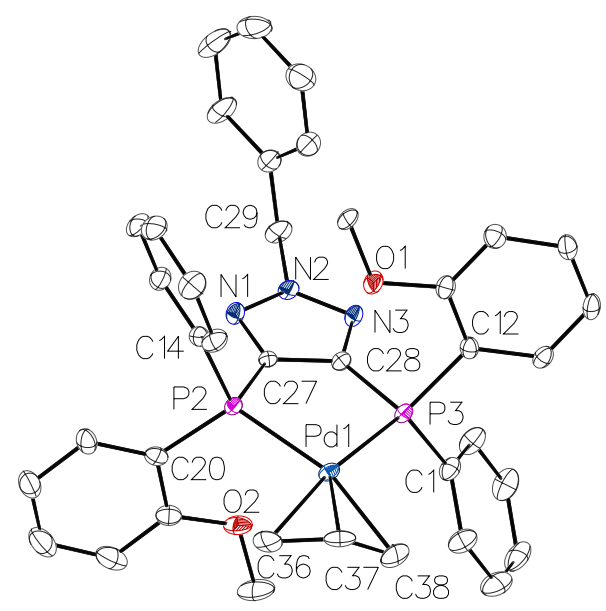

Figure 6. ORTEP view of Pd-complex 14. Thermal ellipsoids are drawn at $50 \%$ probability plot. $\mathrm{H}$ atoms, disordered part and hexafluorophosphate counterion are omitted for clarity.

Finally, the P-chirogenic diphosphinotriazoles 6a-c were used as ligands in the Pd-catalyzed asymmetric allylic substitution reaction of $(E)$-1,3-diphenylprop-2-en-1-yl acetate $15^{[24],[25]}$ (Table 1).

The reaction was firstly carried out using dimethylmalonate as nucleophile with $N, O$-bis(trimethylsilyl)acetamide (BSA) and a catalytic amount of potassium acetate as base in presence of $2 \mathrm{~mol} \%$ of $[\mathrm{Pd}(\mathrm{allyl}) \mathrm{Cl}]_{2}$ and $4 \mathrm{~mol} \%$ of diphosphinotriazoles 6a-c. In dichloromethane, the reactions were completed in two hours at room temperature to selectively afford the mono-allylated compound $\mathbf{1 6 .}$

Table 1. Asymmetric allylic substitution catalyzed by $\mathrm{Pd}$ complexes with diphosphinotriazole ligands $6 a-c .^{[a],[b]}$

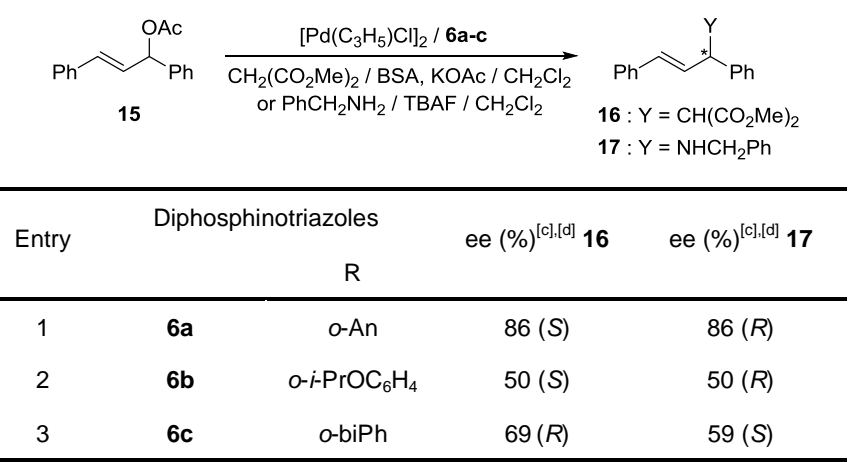

[a] Reactions were carried out in dichloromethane at room temperature and proceeded in completion in $2 \mathrm{~h}$ for $\mathbf{1 6}$ and $18 \mathrm{~h}$ for $\mathbf{1 7}$. [b] Allylic products $\mathbf{1 6}$ and 17 were obtained with yields $>90 \%$ and yields $>85 \%$, respectively. [c] Determined by HPLC on chiral column. [d] Absolute configuration was determined by comparison of the specific rotation with literature values ${ }^{[26]}$

When the catalysis was performed with the P-chirogenic diphosphine $\mathbf{6 a}$ or $\mathbf{6 b}$ bearing respectively, an o-methoxy aryl or an o-isopropoxyaryl substituent on the phosphino groups, the allylated product $\mathbf{1 6}$ was obtained in $(S)$-absolute configuration with $86 \%$ and $50 \%$ ee, respectively (Table 1, entries 1 and 2). In contrast, the use of diphosphinotriazole 6c, having an o-biphenyl group on the phosphorus centres, led to the $(R)$-alkylated product 16 with $69 \%$ ee (Table 1 , entry 3). The allylic substitution of $(E)-1,3$-diphenylprop-2-en-1-yl acetate 15 was also investigated with benzylamine as 
nucleophile in presence of TBAF as additive. The reactions were performed in dichloromethane at room temperature to give in $18 \mathrm{~h}$ the corresponding $(R)$-allylated products 17 with $86 \%$ and $50 \%$ ee when diphosphinotriazoles $6 \mathrm{a}$ and $\mathbf{6 b}$ were used as ligands, respectively (Table 1, entries 1 and 2). In the same conditions, $6 \mathbf{c}$ afforded the allylated product (S)-17 with $59 \%$ ee (Table 1 , entry 3 ).

\section{Conclusion}

A new type of P-chirogenic diphosphines based on a triazole backbone have been stereoselectively synthesized using ephedrine methodology. In order to prove the $P, P$ coordination behavior of these new ligands, a Pd-complex was synthesized and fully characterized by spectroscopic and X-ray diffraction analyses. The usefulness of these $P$ chirogenic diphosphinotriazoles as ligands in asymmetric Pd-catalyzed asymmetric allylic substitution reactions was demonstrated and enantioselectivities up to $86 \%$ were achieved. Further applications of these P-chirogenic diphosphinotriazoles to various asymmetric catalyzed reactions are currently under investigation in our laboratory and will be reported in due course.

\section{Experimental Section}

General: All reactions were carried out using standard Schlenk techniques under an inert gas. Solvents were dried using a MBRAUN SPS 800. Methylene chloride, diethyl ether, ethyl acetate, pentane, petroleum ether, tetrahydrofuran (THF), toluene and methanol were purchased in anhydrous form. Hexane and 2-propanol for HPLC were of chromatographic grade and used without purification. The reagents trimethylsilylacetylene, $n$-BuLi (2.5 M in hexane), DABCO, sulfur, potassium carbonate, benzylbromide, sodium azide, hexachlorodisilane and allylpalladium(II) chloride dimer were purchased from commercial suppliers. $(2 R, 4 S, 5 R)-(+)-3,4-$ dimethyl-2,5diphenyl-1,3,2-oxazaphospholidine-2-borane $\mathbf{1 0}^{[15]}$ as well as (Sp)-(-)-N-methyl-N-[(1R,2S)(1-hydroxy-1-phenyl-2-propyl)]amino-oanisylphenyl-phosphine-borane 11a, ${ }^{[16]}(S p)-(-)-\mathrm{N}$-methyl-N-[(1R,2S)(1-hydroxy-1-phenyl-2-propyl)]amino-(o-isopropoxyphenyl)phenylphosphine-borane $\mathbf{1 1 b}^{[27]}$ and (Sp)-(-)-N-methyl-N-[(1R,2S)(1-hydroxy-1-phenyl-2-propyl)]amino-(o-biphenyl)phenyl-phosphine-borane $11 \mathrm{c}^{[28]}$ were prepared according to published procedure. Flash chromatography was carried out with the indicated solvents using silica gel 60 (60AAC, 35-70 $\mathrm{mm}$; SDS). ${ }^{1} \mathrm{H}$ (and ${ }^{1} \mathrm{H}$ decoupled), ${ }^{13} \mathrm{C}$ and ${ }^{31} \mathrm{P}$ NMR spectra were recorded with Bruker 600 Avance III-HD, Bruker 500 Avance III or Bruker 400 Avance Neo spectrometers at $25^{\circ} \mathrm{C}$, using tetramethylsilane as internal reference for ${ }^{1} \mathrm{H}$ and ${ }^{13} \mathrm{C}$ spectra and $85 \%$ phosphoric acid as external reference for ${ }^{31} \mathrm{P}$ NMR. The signals of ${ }^{13} \mathrm{C}$ NMR spectra were allocated by the J-mod technology. Data are reported in ppm as follows: multiplicity $(s=$ singlet, $d=$ doublet, $t=$ triplet, $q=$ quartet, $m=$ multiplet, br. $s=$ broad signal $)$, coupling constant(s) in Hertz [Hz], integration. HPLC analyses were performed on a Shimadzu chromatograph equipped with a UV detector at $\lambda=254 \mathrm{~nm}$. Mass spectrometry and accurate mass measurements (HRMS) were recorded on a Thermo LTQ Orbitrap XL ESI-MS (ElectroSpray lonization Mass Spectrometry). Melting points were measured with a Kofler melting points apparatus and are uncorrected. Optical rotation values were measured at $20^{\circ} \mathrm{C}$ with a Perkin-Elmer 241 polarimeter at $589 \mathrm{~nm}$ (sodium lamp). Elemental analyses were measured with a precision superior to $0.4 \%$ on a CHNS/-O Thermo Electron Flash EA 1112 Series instrument apparatus. Single Crystal X-ray Diffraction: all experimental data procedure and refinement are detailed in Supplementary Information. Data CCDC-2039650, 2039651 and 2039652 contain the supplementary crystallographic data for this paper of compounds 12a, 6a and 14 respectively. These data can be obtained free of charge from The Cambridge Crystallographic Data Centre via $\underline{w w w . c c d c . c a m . a c . u k / d a t a}$ request/cif

General procedure for the synthesis of P-chirogenic phosphine-sulfides 10: A freshly titrated toluene solution of dry $\mathrm{HCl}(12 \mathrm{mmol})$ was added to aminophosphine-borane $8(2 \mathrm{mmol})$ and the reaction was stirred at room temperature during two hours. The ephedrine hydrochloride was filtered off using a Millipore $4 \mu \mathrm{m}$ filter. The resulting solution of chlorophosphine borane 9 was collected and cooled to $-78^{\circ} \mathrm{C}$. Trimethylsilylacetylenide $(4 \mathrm{mmol})$, previously prepared by reaction between trimethylsilylacetylene $(0.57 \mathrm{~mL}, 4 \mathrm{mmol})$ and $n$ BuLi $\left(2.5 \mathrm{M}\right.$ in hexane) $(1.8 \mathrm{~mL}, 4.4 \mathrm{mmol})$ in $\mathrm{Et}_{2} \mathrm{O}(4 \mathrm{~mL})$ at $-78^{\circ} \mathrm{C}$ during one hour, was added and the resulting mixture was stirred at room temperature during 5 hours. After hydrolysis with water $(10 \mathrm{~mL})$, the mixture was extracted with dichloromethane $(3 \times 10 \mathrm{~mL})$ and the combined organic phases were dried over $\mathrm{MgSO}_{4}$. The solvent was removed under vacuum and the resulting crude product was purified by column chromatography on silica gel using petroleum ether/dichloromethane 1:2 as eluent. The corresponding phosphine borane was dissolved under argon in dry toluene $(5 \mathrm{~mL})$ and DABCO $(0.449 \mathrm{~g}, 4 \mathrm{mmol})$ and sulfur $(0.128 \mathrm{~g}, 4 \mathrm{mmol})$ were successively added. The reaction mixture was stirred at $50{ }^{\circ} \mathrm{C}$ during 3 hours and the solvent was evaporated to give a residue which was purified by column chromatography and/or recrystallization.

(R)-(2-methoxylphenyl)-phenyl-[(2-trimethylsilyl)ethynyl]phosphine sulfide (10a): Reaction was performed using $0.787 \mathrm{~g}$ of aminophosphine-borane 8a. Purification was accomplished by column chromatography on silica gel using petroleum ether/dichloromethane (1:2) as eluent then recrystallization in hexane/dichloromethane. White solid $(0.330 \mathrm{~g}, 48 \%)$. $R_{\mathrm{f}} 0.43$ (petroleum ether/dichloromethane 1:2); m.p. 119-121 ${ }^{\circ} \mathrm{C}$; Enantiomeric excess: $99 \%$ by HPLC analysis (lux $5 \mu$ cellulose-2, $1 \mathrm{~mL} \cdot \mathrm{min}^{-1}$, hexane/2propanol 90:10, $\left.\mathrm{t}_{\mathrm{R}}(R)=8.3 \mathrm{~min}, \mathrm{t}_{\mathrm{R}}(S)=9.9 \mathrm{~min}\right) ;[\alpha]_{\mathrm{D}}-26.3\left(\mathrm{c} 0.3, \mathrm{CHCl}_{3}\right) .{ }^{1} \mathrm{H} \mathrm{NMR}\left(500 \mathrm{MHz}, \mathrm{CDCl}_{3}\right): \delta 0.28(\mathrm{~s}, 9 \mathrm{H}), 3.67(\mathrm{~s}, 3 \mathrm{H}), 6.88$ (dd, $J=6.8,8.5 \mathrm{~Hz}, 1 \mathrm{H}), 7.14$ (tdd, $J=0.9,2.4,7.6 \mathrm{~Hz}, 1 \mathrm{H}), 7.42-7.55(\mathrm{~m}, 4 \mathrm{H}), 7.90-7.95(\mathrm{~m}, 1 \mathrm{H}), 8.23$ (ddd, $J=1.7,7.7,17.9 \mathrm{~Hz}, 1 \mathrm{H})$; $\left\{{ }^{1} \mathrm{H}\right\}^{13} \mathrm{C}$ NMR $(125.8 \mathrm{MHz}, \mathrm{CDCl})$ ): $\delta-0.7,55.3,97.9\left(\mathrm{~d}, J_{C P}=130.9 \mathrm{~Hz}\right), 111.8\left(\mathrm{~d}, J_{C P}=7.1 \mathrm{~Hz}\right), 113.6\left(\mathrm{~d}, J_{C P}=16.3 \mathrm{~Hz}\right), 120.4\left(\mathrm{~d}, J_{C P}=\right.$ $97.6 \mathrm{~Hz}), 120.8\left(\mathrm{~d}, J_{C P}=19.3 \mathrm{~Hz}\right), 128.1\left(\mathrm{~d}, J_{C P}=14.3 \mathrm{~Hz}\right), 130.5\left(\mathrm{~d}, J_{C P}=12.7 \mathrm{~Hz}\right), 131.1\left(\mathrm{~d}, J_{C P}=3.2 \mathrm{~Hz}\right), 134.1\left(\mathrm{~d}, J_{C P}=101.8 \mathrm{~Hz}\right)$, 134.3, 134.4, $160.3\left(\mathrm{~d}, J_{C P}=2.9 \mathrm{~Hz}\right) ;\left\{{ }^{1} \mathrm{H}\right\}^{31} \mathrm{P}$ NMR $\left(202.5 \mathrm{MHz}, \mathrm{CDCl}_{3}\right): \delta+14.6(\mathrm{~s})$. HRMS calcd for $\mathrm{C}_{18} \mathrm{H}_{22} \mathrm{POSSi}[\mathrm{M}+\mathrm{H}]^{+} \mathrm{m} / \mathrm{z}$ 345.08928, found $\mathrm{m} / \mathrm{z}$ 345.08935. Anal. Calcd for $\mathrm{C}_{18} \mathrm{H}_{21}$ POSSi: C, 62.76; $\mathrm{H}, 6.14$; found $\mathrm{C}, 62.56 ; \mathrm{H}, 6.27$.

(R)-(2-isopropoxyphenyl)-phenyl-[(2-trimethylsilyl)ethynyl]phosphine

sulfide (10b): Reaction was performed using $0.842 \mathrm{~g}$ of aminophosphine-borane $\mathbf{8 b}$. Purification was accomplished by column chromatography on silica gel using petroleum ether/dichloromethane $(1: 2)$ as eluent. Visquous colorless oil $(0.417 \mathrm{~g}, 56 \%)$. $\mathrm{R}_{\mathrm{f}} 0.44$ (petroleum ether/dichloromethane 1:2); Enantiomeric excess: 99\% by HPLC analysis (lux 5 $\mu$ amylose-2, $1 \mathrm{~mL} . \mathrm{min}^{-1}$, hexane/2-propanol 
98:2, $\left.\mathrm{t}_{\mathrm{R}}(R)=13.3 \mathrm{~min}, \mathrm{t}_{\mathrm{R}}(S)=15.2 \mathrm{~min}\right) ;[\alpha]_{\mathrm{D}}-79.8\left(\mathrm{c} 0.3, \mathrm{CHCl}_{3}\right) .{ }^{1} \mathrm{H} \mathrm{NMR}\left(500 \mathrm{MHz}, \mathrm{CD}_{2} \mathrm{Cl}_{2}\right): \delta 0.12(\mathrm{~s}, 9 \mathrm{H}), 0.70(\mathrm{~d}, J=5.9 \mathrm{~Hz}, 3 \mathrm{H})$, $0.97(\mathrm{~d}, J=5.9 \mathrm{~Hz}, 3 \mathrm{H}), 4.35$ (hept, $J=5.9 \mathrm{~Hz}, 1 \mathrm{H}), 6.66(\mathrm{dd}, J=6.2,8.3 \mathrm{~Hz}, 1 \mathrm{H}), 6.95$ (tdd, $J=0.8,2.3,7.6 \mathrm{~Hz}, 1 \mathrm{H}), 7.22-7.38(\mathrm{~m}, 4 \mathrm{H})$, 7.61-7.66 (m, 2H), 8.15 (ddd, $J=1.8,7.6,18.1 \mathrm{~Hz}, 1 \mathrm{H}) ;\left\{{ }^{1} \mathrm{H}\right\}^{13} \mathrm{C}$ NMR $\left(151 \mathrm{MHz}, \mathrm{CD}_{2} \mathrm{Cl}_{2}\right): \delta-1.1,20.7,21.1,70.0,97.9\left(\mathrm{~d}, J_{C P}=138.8\right.$ $\mathrm{Hz}), 112.0,113.4\left(\mathrm{~d}, J_{C P}=15.8 \mathrm{~Hz}\right), 119.3\left(\mathrm{~d}, J_{C P}=97.1 \mathrm{~Hz}\right), 119.8\left(\mathrm{~d}, J_{C P}=15.5 \mathrm{~Hz}\right), 128.0\left(J_{C P}=13.6 \mathrm{~Hz}\right), 130.1\left(\mathrm{~d}, J_{C P}=13.6 \mathrm{~Hz}\right)$, $130.9,134.4,135.0\left(\mathrm{~d}, J_{C P}=11.7 \mathrm{~Hz}\right), 135.5\left(\mathrm{~d}, J_{C P}=104.8 \mathrm{~Hz}\right), 158.2 ;\left\{{ }^{1} \mathrm{H}\right\}^{31} \mathrm{P}$ NMR $\left(202.5 \mathrm{MHz}, \mathrm{CD}_{2} \mathrm{Cl}_{2}\right): \delta+14.4(\mathrm{~s})$. HRMS calcd for $\mathrm{C}_{20} \mathrm{H}_{25} \mathrm{POSSiNa}[\mathrm{M}+\mathrm{Na}]^{+} \mathrm{m} / \mathrm{z} 395.10252$, found $\mathrm{m} / \mathrm{z} 395.10237$.

(R)-(o-biphenyl)-phenyl-[(trimethylsilyl)ethynyl]phosphine sulfide (10c): Reaction was performed using $0.878 \mathrm{~g}$ of aminophosphineborane 8c. Purification was accomplished by column chromatography on silica gel using petroleum ether/dichloromethane $(1: 2)$ as eluent. Pale brown sticky solid $(0.336 \mathrm{~g}, 43 \%)$. $\mathrm{R}_{\mathrm{f}} 0.47$ (petroleum ether/dichloromethane 1:2); Enantiomeric excess: $99 \%$ by HPLC analysis (lux $5 \mu$ cellulose-2, $1 \mathrm{~mL} \cdot \mathrm{min}^{-1}$, hexane/2-propanol 98:2, $\mathrm{t}_{\mathrm{R}}(R)=10.2 \mathrm{~min}, \mathrm{t}_{\mathrm{R}}(\mathrm{S})=11.4 \mathrm{~min}$ ); $[\mathrm{\alpha}]_{\mathrm{D}}-18.2\left(\mathrm{c} 0.3, \mathrm{CHCl}_{3}\right) .{ }^{1} \mathrm{H} \mathrm{NMR}$ $\left(600 \mathrm{MHz}, \mathrm{CD}_{2} \mathrm{Cl}_{2}\right): \delta 0.26(\mathrm{~s}, 9 \mathrm{H}), 7.06-7.13(\mathrm{~m}, 4 \mathrm{H}), 7.21-7.31(\mathrm{~m}, 4 \mathrm{H}), 7.40-7.43(\mathrm{~m}, 1 \mathrm{H}), 7.52-7.61(\mathrm{~m}, 4 \mathrm{H}), 8.38$ (ddd, $J=1.3,7.6$, $17.5 \mathrm{~Hz}, 1 \mathrm{H}) ;\left\{\left\{^{1} \mathrm{H}\right\}^{13} \mathrm{C}\right.$ NMR $\left(151 \mathrm{MHz}, \mathrm{CD}_{2} \mathrm{Cl}_{2}\right): \delta-1.2,98.5\left(\mathrm{~d}, J_{C P}=132.2 \mathrm{~Hz}\right), 115.7\left(\mathrm{~d}, J_{C P}=14.8 \mathrm{~Hz}\right), 127.2,127.2\left(\mathrm{~d}, J_{C P}=14.7 \mathrm{~Hz}\right)$, $127.3,128.1\left(\mathrm{~d}, J_{C P}=14.2 \mathrm{~Hz}\right), 130.1,130.7\left(\mathrm{~d}, J_{C P}=12.6 \mathrm{~Hz}\right), 131.1\left(\mathrm{~d}, J_{C P}=3.2 \mathrm{~Hz}\right), 131.6\left(\mathrm{~d}, J_{C P}=3.2 \mathrm{~Hz}\right), 131.8\left(\mathrm{~d}, J_{C P}=95.6 \mathrm{~Hz}\right)$, $132.0\left(\mathrm{~d}, J_{C P}=11.1 \mathrm{~Hz}\right), 133.1\left(\mathrm{~d}, J_{C P}=14.2 \mathrm{~Hz}\right), 133.4\left(\mathrm{~d}, J_{C P}=99.5 \mathrm{~Hz}\right), 139.8\left(\mathrm{~d}, J_{C P}=4.3 \mathrm{~Hz}\right), 145.6\left(\mathrm{~d}, J_{C P}=10.9 \mathrm{~Hz}\right) ;\left\{{ }^{1} \mathrm{H}\right\}^{31} \mathrm{P}$ NMR $\left(243 \mathrm{MHz}, \mathrm{CD}_{2} \mathrm{Cl}_{2}\right.$ ): $\delta+18.5$ (s). HRMS calcd for $\mathrm{C}_{23} \mathrm{H}_{24} \mathrm{PSSi}[\mathrm{M}+\mathrm{H}]^{+} \mathrm{m} / \mathrm{z} 391.11001$, found $\mathrm{m} / \mathrm{z} 391.11055$. Anal. Calcd for $\mathrm{C}_{23} \mathrm{H}_{23}$ PSSi: C, 70.73; H, 5.94; found C, 70.84; H, 5.99 .

General procedure for the synthesis of P-chirogenic phosphine-sulfides 11: To a solution of phosphine sulfide 10a-c $(0.5 \mathrm{mmol})$ in a mixture methanol/THF (1:1) $(3 \mathrm{~mL})$ was added potassium carbonate $(0.5 \mathrm{mmol})$. The reaction mixture was stirred at room temperature during 15 minutes and the solvent was evaporated to dryness. Water $(5 \mathrm{~mL})$ and dichloromethane $(5 \mathrm{~mL})$ were added and the aqueous phase was extracted twice with dichloromethane $(2 \times 5 \mathrm{~mL})$. The organic phases were dried over $\mathrm{MgSO}_{4}$, filtered and evaporated to give a residue which was purified by column chromatography.

(S)-ethynyl-(2-methoxylphenyl)-phenylphosphine sulfide (11a): Reaction was performed using $0.172 \mathrm{~g}$ of phosphine-sulfide 10a. Purification was accomplished by column chromatography on silica gel using petroleum ether/dichloromethane (1:3) as eluent. White solid $\left(0.117 \mathrm{~g}, 86 \%\right.$ ). $\mathrm{R}_{\mathrm{f}} 0.40$ (petroleum ether/dichloromethane $1: 3$ ); $\mathrm{mp} 119-121^{\circ} \mathrm{C}$; Enantiomeric excess: $99 \%$ by HPLC analysis (lux $5 \mu$ cellulose-1, $1 \mathrm{~mL} \cdot \mathrm{min}^{-1}$, hexane/2-propanol 90:10, $\left.\mathrm{t}_{\mathrm{R}}(R)=15.7 \mathrm{~min}, \mathrm{t}_{\mathrm{R}}(S)=17.3 \mathrm{~min}\right)$; $[\alpha]_{\mathrm{D}}-2.6\left(\mathrm{c} 0.3, \mathrm{CHCl}_{3}\right) .{ }^{1} \mathrm{H} \mathrm{NMR}(500 \mathrm{MHz}$, $\left.\mathrm{CDCl}_{3}\right): \delta 3.37(\mathrm{~d}, J=10.1 \mathrm{~Hz}, 1 \mathrm{H}), 3.66(\mathrm{~s}, 3 \mathrm{H}), 6.88-6.91(\mathrm{~m}, 1 \mathrm{H}), 7.16(\mathrm{tdd}, J=0.6,2.3,7.5 \mathrm{~Hz}, 1 \mathrm{H}), 7.43-7.57(\mathrm{~m}, 4 \mathrm{H}), 7.90-7.95(\mathrm{~m}$, 2H), 8.24 (ddd, $J=1.7,7.6,18.0 \mathrm{~Hz}, 1 \mathrm{H}) ;\left\{{ }^{1} \mathrm{H}\right\}^{13} \mathrm{C} N \mathrm{NMR}\left(125.8 \mathrm{MHz}, \mathrm{CDCl}_{3}\right): \delta 55.5,78.4\left(\mathrm{~d}, J_{C P}=145.2 \mathrm{~Hz}\right), 92.8\left(\mathrm{~d}, J_{C P}=25.2 \mathrm{~Hz}\right)$, $111.9\left(\mathrm{~d}, J_{C P}=5.6 \mathrm{~Hz}\right), 119.6\left(\mathrm{~d}, J_{C P}=98.7 \mathrm{~Hz}\right), 121.0\left(\mathrm{~d}, J_{C P}=14.6 \mathrm{~Hz}\right), 128.2\left(\mathrm{~d}, J_{C P}=14.1 \mathrm{~Hz}\right), 130.4\left(\mathrm{~d}, J_{C P}=12.7 \mathrm{~Hz}\right), 131.4(\mathrm{~d}$, $\left.J_{C P}=2.8 \mathrm{~Hz}\right), 133.8\left(\mathrm{~d}, J_{C P}=102.8 \mathrm{~Hz}\right), 134.4(\mathrm{~d}, J=12.0 \mathrm{~Hz}), 134.7\left(\mathrm{~d}, J_{C P}=2.8 \mathrm{~Hz}\right), 160.3\left(\mathrm{~d}, J_{C P}=2.4 \mathrm{~Hz}\right) ;\left\{{ }^{1} \mathrm{H}{ }^{31} \mathrm{P}\right.$ NMR $(202.5$ $\mathrm{MHz}, \mathrm{CDCl}_{3}$ ): $\delta+16.4$ (s). HRMS calcd for $\mathrm{C}_{15} \mathrm{H}_{14} \mathrm{PSO}[\mathrm{M}+\mathrm{H}]^{+} \mathrm{m} / \mathrm{z} 273.04975$, found $\mathrm{m} / z$ 273.04904. Anal. Calcd for $\mathrm{C}_{15} \mathrm{H}_{13} \mathrm{PSO}: \mathrm{C}$, 66.16; H, 4.81; found $\mathrm{C}, 66.01 ; \mathrm{H}, 4.93$.

(S)-ethynyl-(2-isopropoxyphenyl)-phenylphosphine sulfide (11b): Reaction was performed using $0.186 \mathrm{~g}$ of phosphine-sulfide $10 \mathrm{~b}$. Purification was accomplished by column chromatography on silica gel using petroleum ether/dichloromethane (1:2) as eluent. White solid $\left(0.126 \mathrm{~g}, 84 \%\right.$ ). $\mathrm{R}_{\mathrm{f}} 0.42$ (petroleum ether/dichloromethane $1: 2$ ); mp $98-100^{\circ} \mathrm{C}$; Enantiomeric excess: $99 \%$ by HPLC analysis (lux $5 \mu$ cellulose-2, $1.0 \mathrm{~mL} \cdot \mathrm{min}^{-1}$, hexane/2-propanol 95:5, $\left.\mathrm{t}_{\mathrm{R}}(R)=33.5 \mathrm{~min}, \mathrm{t}_{\mathrm{R}}(S)=38.2 \mathrm{~min}\right)$; $[\alpha]_{\mathrm{D}}-39.3\left(\mathrm{c} 0.3, \mathrm{CHCl}_{3}\right)$. ${ }^{1} \mathrm{H} \mathrm{NMR}(600 \mathrm{MHz}$, $\left.\mathrm{CD}_{2} \mathrm{Cl}_{2}\right): \delta 0.95(\mathrm{~d}, J=6.3 \mathrm{~Hz}, 3 \mathrm{H}), 1.15(\mathrm{~d}, J=6.3 \mathrm{~Hz}, 3 \mathrm{H}), 3.45(\mathrm{~d}, J=10.6 \mathrm{~Hz}, 1 \mathrm{H}), 4.57$ (hept, $\left.J=6.3 \mathrm{~Hz}, 1 \mathrm{H}\right), 6.89(\mathrm{dd}, J=6.3,7.9$ $\mathrm{Hz}, 1 \mathrm{H}), 7.16(\mathrm{td}, J=24,7.5 \mathrm{~Hz}, 1 \mathrm{H}), 7.45-7.59(\mathrm{~m}, 4 \mathrm{H}), 7.85-7.89(\mathrm{~m}, 2 \mathrm{H}), 8.34$ (ddd, $J=1.6,7.8,18.0 \mathrm{~Hz}, 1 \mathrm{H}) ;\left\{{ }^{1} \mathrm{H}\right\}^{13} \mathrm{C} \mathrm{NMR}(151$ $\left.\mathrm{MHz}, \mathrm{CD}_{2} \mathrm{Cl}_{2}\right): \delta 20.7,21.3,70.3,78.4\left(\mathrm{~d}, J_{C P}=149.6 \mathrm{~Hz}\right), 92.6\left(\mathrm{~d}, J_{C P}=25.7 \mathrm{~Hz}\right), 112.1\left(\mathrm{~d}, J_{C P}=6.3 \mathrm{~Hz}\right), 118.9\left(\mathrm{~d}, J_{C P}=98.2 \mathrm{~Hz}\right)$, $119.9\left(\mathrm{~d}, J_{C P}=14.6 \mathrm{~Hz}\right), 128.1\left(\mathrm{~d}, J_{C P}=13.8 \mathrm{~Hz}\right), 130.1\left(\mathrm{~d}, J_{C P}=12.1 \mathrm{~Hz}\right), 131.2\left(\mathrm{~d}, J_{C P}=2.6 \mathrm{~Hz}\right), 134.6\left(\mathrm{~d}, J_{C P}=1.7 \mathrm{~Hz}\right), 134.8\left(\mathrm{~d}, J_{C P}\right.$ $=11.2 \mathrm{~Hz}), 134.9\left(\mathrm{~d}, J_{C P}=104.4 \mathrm{~Hz}\right), 158.3 ;\left\{{ }^{1} \mathrm{H}\right\}^{31} \mathrm{P} \mathrm{NMR}\left(243 \mathrm{MHz}, \mathrm{CD}_{2} \mathrm{Cl}_{2}\right): \delta+16.2(\mathrm{~s})$. HRMS calcd for $\mathrm{C}_{17} \mathrm{H}_{17} \mathrm{POSNa}[\mathrm{M}+\mathrm{Na}]^{+} \mathrm{m} / \mathrm{z}$ 323.06299, found $\mathrm{m} / z$ 323.06249. Anal. Calcd for $\mathrm{C}_{17} \mathrm{H}_{17}$ PSO: C, 67.98; H, 5.71; found C, 68.29; H, 5.85 .

(S)-(o-biphenyl)-ethynyl-phenylphosphine sulfide (11c): Reaction was performed using $0.195 \mathrm{~g}$ of phosphine-sulfide 10c. Purification was accomplished by column chromatography on silica gel using petroleum ether/dichloromethane (1:2) as eluent. White sticky solid $(0.137 \mathrm{~g}, 86 \%) ; R_{\mathrm{f}} 0.44$ (petroleum ether/dichloromethane 1:2); Enantiomeric excess: $99 \%$ by HPLC analysis (lux $5 \mu$ cellulose-1, 0.7 $\mathrm{mL} \cdot \mathrm{min}^{-1}$, hexane/2-propanol 98:2, $\left.\mathrm{t}_{\mathrm{R}}(R)=30.2 \mathrm{~min}, \mathrm{t}_{\mathrm{R}}(S)=31.5 \mathrm{~min}\right) ;[\mathrm{\alpha}]_{\mathrm{D}}+24.7\left(\mathrm{c} 0.3, \mathrm{CHCl}_{3}\right) .{ }^{1} \mathrm{H}$ NMR $\left(500 \mathrm{MHz}, \mathrm{CD}_{2} \mathrm{Cl}_{2}\right): \delta 3.28(\mathrm{~d}$, $J=9.0 \mathrm{~Hz}, 1 \mathrm{H}), 6.99-7.05(\mathrm{~m}, 4 \mathrm{H}), 7.13-7.24(\mathrm{~m}, 4 \mathrm{H}), 7.33-7.37(\mathrm{~m}, 1 \mathrm{H}), 7.45-7.56(\mathrm{~m}, 4 \mathrm{H}), 8.28(\mathrm{ddd}, J=1.3,7.5,17.3 \mathrm{~Hz}, 1 \mathrm{H})$; $\left\{{ }^{1} \mathrm{H}\right\}^{13} \mathrm{C}$ NMR $\left(125.8 \mathrm{MHz}, \mathrm{CD}_{2} \mathrm{Cl}_{2}\right): \delta 78.8\left(\mathrm{~d}, J_{C P}=144.7 \mathrm{~Hz}\right), 94.3\left(\mathrm{~d}, J_{C P}=23.6 \mathrm{~Hz}\right), 127.2,127.3\left(\mathrm{~d}, J_{C P}=14.0 \mathrm{~Hz}\right), 127.4,128.2(\mathrm{~d}$, $\left.J_{C P}=14.0 \mathrm{~Hz}\right), 130.1,130.8\left(\mathrm{~d}, J_{C P}=12.4 \mathrm{~Hz}\right), 131.4\left(\mathrm{~d}, J_{C P}=3.3 \mathrm{~Hz}\right), 131.7\left(\mathrm{~d}, J_{C P}=97.5 \mathrm{~Hz}\right), 131.8\left(\mathrm{~d}, J_{C P}=2.8 \mathrm{~Hz}\right), 132.1\left(\mathrm{~d}, J_{C P}=\right.$ $10.5 \mathrm{~Hz}), 132.5\left(\mathrm{~d}, J_{C P}=97.5 \mathrm{~Hz}\right), 132.8\left(\mathrm{~d}, J_{C P}=13.8 \mathrm{~Hz}\right), 139.6\left(\mathrm{~d}, J_{C P}=3.6 \mathrm{~Hz}\right), 145.6\left(\mathrm{~d}, J_{C P}=12.6 \mathrm{~Hz}\right) ;\left\{{ }^{1} \mathrm{H}\right\}^{31} \mathrm{P} \mathrm{NMR}(202.5 \mathrm{MHz}$, $\left.\mathrm{CD}_{2} \mathrm{Cl}_{2}\right): \delta+20.5$ (s). HRMS calcd for $\mathrm{C}_{20} \mathrm{H}_{15} \mathrm{PSNa}[\mathrm{M}+\mathrm{Na}]^{+} \mathrm{m} / \mathrm{z} 341.05243$, found $\mathrm{m} / z 341.05158$.

General procedure for the synthesis of P-chirogenic bis(thiophosphinyl)acetylenes 12: A freshly titrated toluene solution of dry $\mathrm{HCl}(6 \mathrm{mmol})$ was added to aminophosphine borane $8(1 \mathrm{mmol})$ and the reaction was stirred at room temperature during two hours. The ephedrine hydrochloride was filtered off using a Millipore $4 \mu \mathrm{m}$ filter. The resulting solution of chlorophosphine borane 9 was collected and cooled to $-78^{\circ} \mathrm{C}$. Thiophosphine acetylenide $(0.67 \mathrm{mmol})$, previously prepared by reaction between phosphine-sulfide $11(0.67$ $\mathrm{mmol})$ and $n$-BuLi $\left(2.5 \mathrm{M}\right.$ in hexane) $(0.30 \mathrm{~mL}, 0.74 \mathrm{mmol})$ in $\mathrm{Et}_{2} \mathrm{O} / \mathrm{THF}(1.5 \mathrm{~mL} / 1.5 \mathrm{~mL})$ at $-78^{\circ} \mathrm{C}$ during one hour, was added and the resulting mixture was stirred at room temperature during 5 hours. After hydrolysis with water $(10 \mathrm{~mL})$, the mixture was extracted with dichloromethane $(3 \times 10 \mathrm{~mL})$ and the combined organic phases were dried over $\mathrm{MgSO}_{4}$. The solvent was removed under vacuum and the resulting crude product was purified by column chromatography on silica gel using petroleum ether/dichloromethane 1:2 as eluent. The intermediate phosphine-sulfide phosphine-borane was dissolved under argon in dry toluene $(4 \mathrm{~mL})$ and DABCO $(0.157 \mathrm{~g}, 1.4 \mathrm{mmol})$ and sulfur $(0.045 \mathrm{~g}, 1.4 \mathrm{mmol})$ were successively added. The reaction mixture was stirred at $50{ }^{\circ} \mathrm{C}$ overnight and the solvent was evaporated to give a residue which was purified by column chromatography and recrystallization.

$(R, R)$-Bis-[(2-methoxyphenyl)-phenyl-thiophosphinyl]acetylene (12a): Reaction was performed using $0.393 \mathrm{~g}$ of aminophosphineborane $8 \mathrm{a}$ and $0.182 \mathrm{~g}$ of phosphine-sulfide 11a. Purification was accomplished by column chromatography on silica gel using dichloromethane as eluent then recrystallization in hexane/dichloromethane. White solid $(0.177 \mathrm{~g}, 51 \%) . R_{\mathrm{f}} 0.49$ (dichloromethane); $\mathrm{mp}$ $168-170^{\circ} \mathrm{C}$; $[\alpha]_{\mathrm{D}}-81.0\left(\mathrm{c} 0.3, \mathrm{CHCl}_{3}\right)$ for $\mathrm{ee}=99 \% .{ }^{1} \mathrm{H}$ NMR $\left(500 \mathrm{MHz}, \mathrm{CDCl}_{3}\right)$ : $\delta 3.62(\mathrm{~s}, 6 \mathrm{H}), 6.86(\mathrm{dd}, J=5.8,8.3 \mathrm{~Hz}, 2 \mathrm{H}), 7.14$ (tdd, $J$ 
$=0.8,2.4,7.6 \mathrm{~Hz}, 2 \mathrm{H}), 7.42-7.56(\mathrm{~m}, 8 \mathrm{H}), 7.88-7.94(\mathrm{~m}, 4 \mathrm{H}), 8.18$ (ddd, $J=1.7,7.7,18.0 \mathrm{~Hz}, 2 \mathrm{H}) ;\left\{{ }^{1} \mathrm{H}\right\}^{13} \mathrm{C} \mathrm{NMR}\left(125.8 \mathrm{MHz}, \mathrm{CDCl}_{3}\right): \delta$ 55.8, $96.7\left(\mathrm{dd}, J_{C P}=16.6,133.7 \mathrm{~Hz}\right), 111.7\left(\mathrm{t}, J_{C P}=3.1 \mathrm{~Hz}\right), 119.0\left(\mathrm{~d}, J_{C P}=98.2 \mathrm{~Hz}\right), 121.0-121.1(\mathrm{~m}), 128.4\left(\mathrm{~d}, J_{C P}=14.5 \mathrm{~Hz}\right), 128.4$ (t, $\left.J_{C P}=9.9 \mathrm{~Hz}\right), 130.5\left(\mathrm{~d}, J_{C P}=13.2 \mathrm{~Hz}\right), 130.5\left(\mathrm{t}, J_{C P}=9.2 \mathrm{~Hz}\right), 131.6,133.5\left(\mathrm{~d}, J_{C P}=103.6 \mathrm{~Hz}\right), 134.4-134.5(\mathrm{~m}), 134.9,160.4$; $\left\{{ }^{1} \mathrm{H}\right\}^{31} \mathrm{P}$ NMR $\left(202.5 \mathrm{MHz}, \mathrm{CDCl}_{3}\right)$ : $\delta+16.7$ (s). HRMS calcd for $\mathrm{C}_{28} \mathrm{H}_{25} \mathrm{P}_{2} \mathrm{O}_{2} \mathrm{~S}_{2}[\mathrm{M}+\mathrm{H}]^{+} \mathrm{m} / z$ 519.07657, found $\mathrm{m} / z$ 519.07589. Anal. Calcd for $\mathrm{C}_{28} \mathrm{H}_{24} \mathrm{P}_{2} \mathrm{O}_{2} \mathrm{~S}_{2}$ : C, 64.85; $\mathrm{H}, 4.67$; found $\mathrm{C}, 64.58 ; \mathrm{H}, 4.78$.

$(\boldsymbol{R}, \boldsymbol{R})$-Bis-[(2-isopropoxyphenyl)-phenyl-thiophosphinyl]acetylene (12b): Reaction was performed using $0.421 \mathrm{~g}$ of aminophosphine-borane $\mathbf{8 b}$ and $0.201 \mathrm{~g}$ of phosphine-sulfide $\mathbf{1 1 b}$. Purification was accomplished by column chromatography on silica gel using petroleum ether/dichloromethane $(1: 3)$ as eluent. White solid $(0.181 \mathrm{~g}, 47 \%) . R_{\mathrm{f}} 0.38$ (petroleum ether/dichloromethane $1: 2$ ); $\mathrm{mp} 222-224^{\circ} \mathrm{C}$; [a]D -172.1 (c 0.3, $\left.\mathrm{CHCl}_{3}\right)$ for ee $=99 \% .{ }^{1} \mathrm{H} \mathrm{NMR}\left(600 \mathrm{MHz}, \mathrm{CD}_{2} \mathrm{Cl}_{2}\right): \delta 0.88(\mathrm{~d}, J=6.2 \mathrm{~Hz}, 6 \mathrm{H}), 0.97(\mathrm{~d}, J=6.2 \mathrm{~Hz}, 6 \mathrm{H})$, 4.46 (hept, $J=6.2 \mathrm{~Hz}, 2 \mathrm{H}$ ), 6.84 (dd, $J=6.1,8.2 \mathrm{~Hz}, 2 \mathrm{H}$ ), 7.13 (td, $J=1.8,7.5 \mathrm{~Hz}, 2 \mathrm{H}$ ), 7.46-7.57 (m, 8H), 7.85-7.89 (m, $4 \mathrm{H}$ ), 8.30 (ddd, $J=1.8,7.7,18.2 \mathrm{~Hz}, 2 \mathrm{H}) ;\left\{{ }^{1} \mathrm{H}\right\}^{13} \mathrm{C} \mathrm{NMR}\left(151 \mathrm{MHz}, \mathrm{CD}_{2} \mathrm{Cl}_{2}\right): \delta 20.7(2 \mathrm{~s}), 70.4,96.7$ (dd, $\left.J_{C P}=16.0,131.8 \mathrm{~Hz}\right), 112.1\left(\mathrm{t}, J_{C P}=2.8 \mathrm{~Hz}\right)$, $118.0\left(\mathrm{~d}, J_{C P}=98.6 \mathrm{~Hz}\right), 120.0\left(\mathrm{~d}, J_{C P}=15.4 \mathrm{~Hz}\right), 120.0\left(\mathrm{t}, J_{C P}=9.4 \mathrm{~Hz}\right), 128.3\left(\mathrm{~d}, J_{C P}=14.6 \mathrm{~Hz}\right), 128.3\left(\mathrm{~d}, J_{C P}=9.6 \mathrm{~Hz}\right), 130.1\left(\mathrm{~d}, J_{C P}\right.$ $=13.0 \mathrm{~Hz}), 130.1\left(\mathrm{t}, J_{C P}=8.0 \mathrm{~Hz}\right), 131.4,134.4,134.8,134.9-135.0(\mathrm{~m}), 158.4 ;\left\{{ }^{1} \mathrm{H}{ }^{31} \mathrm{P} \mathrm{NMR}\left(243 \mathrm{MHz}, \mathrm{CD}_{2} \mathrm{Cl}_{2}\right): \delta+16.7(\mathrm{~s})\right.$. HRMS calcd for $\mathrm{C}_{32} \mathrm{H}_{32} \mathrm{O}_{2} \mathrm{P}_{2} \mathrm{~S}_{2} \mathrm{Na}[\mathrm{M}+\mathrm{Na}]^{+} \mathrm{m} / z$ 597.1211, found $\mathrm{m} / \mathrm{z}$ 597.1206. Anal. Calcd for $\mathrm{C}_{32} \mathrm{H}_{32} \mathrm{O}_{2} \mathrm{P}_{2} \mathrm{~S}_{2}: \mathrm{C}, 66.88 ; \mathrm{H}, 5.61$; found $\mathrm{C}, 66.62$; H, 5.55 .

$(\boldsymbol{R}, \boldsymbol{R})$-Bis-[(o-biphenyl)-phenyl-thiophosphinyl]acetylene (12c): Reaction was performed using $0.439 \mathrm{~g}$ of aminophosphine-borane $8 \mathrm{c}$ and $0.213 \mathrm{~g}$ of phosphine-sulfide 11c. Purification was accomplished by column chromatography on silica gel using petroleum ether/dichloromethane (1:2) as eluent. White solid $(0.172 \mathrm{~g}, 42 \%)$. $R_{f} 0.47$ (petroleum ether/dichloromethane 1:2); mp 208-210 ${ }^{\circ} \mathrm{C} ;[\alpha]_{D}$ $103.3\left(\mathrm{c} 0.3, \mathrm{CHCl}_{3}\right)$ for ee $=99 \% .{ }^{1} \mathrm{H} \mathrm{NMR}\left(600 \mathrm{MHz}, \mathrm{CD}_{2} \mathrm{Cl}_{2}\right)$ : $\delta 6.99-7.00(\mathrm{~m}, 8 \mathrm{H}), 7.06-7.08(\mathrm{~m}, 2 \mathrm{H}), 7.22-7.25(\mathrm{~m}, 6 \mathrm{H}), 7.35-7.42(\mathrm{~m}$, $6 \mathrm{H}$ ), 7.56-7.64 (m, 4H), 8.30 (dd, $J=8.0,17.6 \mathrm{~Hz}, 2 \mathrm{H}) ;\left\{{ }^{1} \mathrm{H}\right\}^{13} \mathrm{C}$ NMR $\left(151 \mathrm{MHz}, \mathrm{CD}_{2} \mathrm{Cl}_{2}\right.$ ): $\delta 99.3$ (dd, $\left.J_{C P}=13.5,126.0 \mathrm{~Hz}\right), 127.3,127.4$ $(2 \mathrm{~s}), 128.3\left(\mathrm{~d}, J_{C P}=14.6 \mathrm{~Hz}\right), 128.3,130.0,130.6\left(\mathrm{~d}, J_{C P}=12.5 \mathrm{~Hz}\right), 130.6,130.7\left(\mathrm{~d}, J_{C P}=98.0 \mathrm{~Hz}\right), 131.3,132.0\left(\mathrm{~d}, J_{C P}=98.0 \mathrm{~Hz}\right)$, 132.0-132.1 (m), $133.3\left(\mathrm{~d}, J_{C P}=14.2 \mathrm{~Hz}\right), 133.3,139.1$ (br. t, $\left.J_{C P}=1.8 \mathrm{~Hz}\right), 145.9\left(\mathrm{~d}, J_{C P}=11.0 \mathrm{~Hz}\right), 145.9 ;\left\{{ }^{1} \mathrm{H}\right\}^{31} \mathrm{P} \mathrm{NMR}(243 \mathrm{MHz}$, $\mathrm{CD}_{2} \mathrm{Cl}_{2}$ ): $\delta+21.6$ (s). HRMS calcd for $\mathrm{C}_{38} \mathrm{H}_{28} \mathrm{P}_{2} \mathrm{~S}_{2} \mathrm{Na}[\mathrm{M}+\mathrm{Na}]^{+} \mathrm{m} / \mathrm{z} 633.09999$, found $\mathrm{m} / \mathrm{z}$ 633.10064. Anal. Calcd for $\mathrm{C}_{38} \mathrm{H}_{28} \mathrm{P}_{2} \mathrm{~S}_{2}: \mathrm{C}_{\text {, }}$ 74.74; H, 4.62; found C, 74.88; H, 4.85 .

General procedure for the synthesis of P-chirogenic 4,5-bis(thiophosphinyl)-1,2,3-triazoles 13: To a solution of bis(thiophosphanyl)acetylene $12(0.30 \mathrm{mmol})$ in $\operatorname{DMF}(1 \mathrm{~mL})$ was added $\mathrm{NaN}_{3}(0.043 \mathrm{~g}, 0.66 \mathrm{mmol})$. The resulting mixture was stirred at $50^{\circ} \mathrm{C}$ during $16 \mathrm{~h}$ then cooled to room temperature. $\mathrm{K}_{2} \mathrm{CO}_{3}(0.137 \mathrm{~g}, 0.99 \mathrm{mmol})$ and benzyl bromide $(0.12 \mathrm{~mL}, 0.99 \mathrm{mmol})$ were successively added and the mixture was stirred at room temperature during $5 \mathrm{~h}$. After hydrolysis with water (10 $\mathrm{mL})$, the mixture was extracted with dichloromethane $(3 \times 10 \mathrm{~mL})$ and the combined organic phases were dried over $\mathrm{MgSO}_{4}$. The solvent was removed under vacuum and the resulting crude product was purified by column chromatography.

$(R, R)$-(2-benzyl)-4,5-bis-[(2-methoxyphenyl)-phenyl-thiophosphinyl]-2H-1,2,3-triazole (13a): Reaction was performed using $0.155 \mathrm{~g}$ of bis(thiophosphinyl)acetylene 12a. Purification was accomplished by column chromatography on silica gel using dichloromethane as eluent. White solid $\left(0.15 \mathrm{~g}, 79 \%\right.$ ). $\mathrm{R}_{\mathrm{f}} 0.14$ (dichloromethane); $\mathrm{mp} 205-207^{\circ} \mathrm{C}$; Enantiomeric excess: $99 \%$ by HPLC analysis (lux $5 \mu$ cellulose-1, $1.0 \mathrm{~mL} \cdot \mathrm{min}^{-1}$, hexane/2-propanol 95:5, $\left.\mathrm{t}_{\mathrm{R}}(R, R)=40.9 \mathrm{~min}, \mathrm{t}_{\mathrm{R}}(S, S)=53.8 \mathrm{~min}, \mathrm{t}_{\mathrm{R}}(R, S)=64.1 \mathrm{~min}\right)$; $[\alpha]_{\mathrm{D}}+74.3$ (c 0.3 , $\left.\mathrm{CHCl}_{3}\right) .{ }^{1} \mathrm{H}$ NMR $\left(600 \mathrm{MHz}, \mathrm{CD}_{2} \mathrm{Cl}_{2}\right): \delta 3.35(\mathrm{~s}, 6 \mathrm{H}), 5.62(2 \mathrm{ABX}$ syst, $J=14.6,20.1 \mathrm{~Hz}, 2 \mathrm{H}), 6.81$ (dd, $\left.J=5.9,8.3 \mathrm{~Hz}, 2 \mathrm{H}\right), 7.05$ (tdd, $J$ $=0.9,2.1,7.7 \mathrm{~Hz}, 2 \mathrm{H}), 7.28-7.30(\mathrm{~m}, 2 \mathrm{H}), 7.37-7.41(\mathrm{~m}, 7 \mathrm{H}), 7.47-7.50(\mathrm{~m}, 2 \mathrm{H}), 7.54-7.57(\mathrm{~m}, 2 \mathrm{H}), 7.72(\mathrm{ddd}, J=1.8,7.7,17.5 \mathrm{~Hz}, 2 \mathrm{H})$, 7.85-7.88 (m, 4H); $\left\{{ }^{1} \mathrm{H}\right\}^{13} \mathrm{C}$ NMR $\left(151 \mathrm{MHz}, \mathrm{CD}_{2} \mathrm{Cl}_{2}\right): \delta 54.8,59.0,111.4\left(\mathrm{~d}, J_{C P}=7.8 \mathrm{~Hz}\right), 119.5\left(\mathrm{~d}, J_{C P}=90.9 \mathrm{~Hz}\right), 120.6(\mathrm{~d}, J=13.6$ $\mathrm{Hz}), 127.4\left(\mathrm{~d}, J_{C P}=16.4 \mathrm{~Hz}\right), 128.0,128.5,128.7,130.9,132.4\left(\mathrm{~d}, J_{C P}=10.9 \mathrm{~Hz}\right), 133.1\left(\mathrm{~d}, J_{C P}=92.9 \mathrm{~Hz}\right), 134.0,134.9,135.1\left(\mathrm{~d}, J_{C P}=\right.$ $10.9 \mathrm{~Hz}), 146.5\left(\mathrm{dd}, J_{C P}=19.4,115.2 \mathrm{~Hz}\right), 160.4 ;\left\{{ }^{1} \mathrm{H}\right\}^{31} \mathrm{P}$ NMR $\left(243 \mathrm{MHz}, \mathrm{CD}_{2} \mathrm{Cl}_{2}\right): \delta+26.5$ (s). HRMS calcd for $\mathrm{C}_{35} \mathrm{H}_{31} \mathrm{~N}_{3} \mathrm{O}_{2} \mathrm{P}_{2} \mathrm{~S}_{2} \mathrm{Na}$ $[\mathrm{M}+\mathrm{Na}]^{+} \mathrm{m} / \mathrm{z}$ 674.12251, found $\mathrm{m} / \mathrm{z}$ 674.12296. Anal. Calcd for $\mathrm{C}_{35} \mathrm{H}_{31} \mathrm{~N}_{3} \mathrm{O}_{2} \mathrm{P}_{2} \mathrm{~S}_{2}: \mathrm{C}, 64.50 ; \mathrm{H}, 4.79$; found $\mathrm{C}, 64.78 ; \mathrm{H}, 4.73$.

$(R, R)$-(2-benzyl)-4,5-bis-[(2-isopropoxyphenyl)-phenyl-thiophosphi- nyl]-2H-1,2,3-triazole (13b): Reaction was performed using $0.172 \mathrm{~g}$ of bis(thiophosphinyl)acetylene 12b. Purification was accomplished by column chromatography on silica gel using dichloromethane as eluent. White solid $(0.13 \mathrm{~g}, 61 \%)$. $R_{\mathrm{f}} 0.45$ (dichloromethane); $\mathrm{mp} 182-184^{\circ} \mathrm{C}$; Enantiomeric excess: $99 \%$ by HPLC analysis (lux $5 \mu$ cellulose-1, $1.0 \mathrm{~mL} \cdot \mathrm{min}^{-1}$, hexane/2-propanol 95:5, $\left.\mathrm{t}_{\mathrm{R}}(R, R)=13.1 \mathrm{~min}, \mathrm{t}_{\mathrm{R}}(S, S)=15.1 \mathrm{~min}, \mathrm{t}_{\mathrm{R}}(R, S)=16.3 \mathrm{~min}\right) ;[\mathrm{\alpha}]_{\mathrm{D}}$ $+142.4\left(\mathrm{c} 0.3, \mathrm{CHCl}_{3}\right) .{ }^{1} \mathrm{H}$ NMR $\left(500 \mathrm{MHz}, \mathrm{CD}_{2} \mathrm{Cl}_{2}\right): \delta 0.52(\mathrm{~d}, J=6.0 \mathrm{~Hz}, 6 \mathrm{H}), 0.79(\mathrm{~d}, J=6.0 \mathrm{~Hz}, 6 \mathrm{H}), 4.23(\mathrm{hept}, J=6.0 \mathrm{~Hz}, 2 \mathrm{H}), 5.43$ (2 AB syst, $J=14.3 \mathrm{~Hz}, 2 \mathrm{H}$ ), 6.64 (dd, $J=6.2,8.6 \mathrm{~Hz}, 2 \mathrm{H}$ ), 6.87 (tdd, $J=0.7,2.1,7.5 \mathrm{~Hz}, 2 \mathrm{H}$ ), 7.21-7.42 (m, 13H), 7.59 (ddd, $J=1.6$, $7.5,17.4 \mathrm{~Hz}, 2 \mathrm{H}), 7.75-7.80(\mathrm{~m}, 4 \mathrm{H}) ;\left\{{ }^{1} \mathrm{H}\right\}^{13} \mathrm{C} N \mathrm{NMR}\left(151 \mathrm{MHz}, \mathrm{CD}_{2} \mathrm{Cl}_{2}\right): \delta 20.5,21.3,59.0,69.8,111.7\left(\mathrm{~d}, J_{C P}=6.4 \mathrm{~Hz}\right), 118.2\left(\mathrm{~d}, J_{C P}=\right.$ $92.0 \mathrm{~Hz}), 119.6\left(\mathrm{~d}, J_{C P}=14.6 \mathrm{~Hz}\right), 127.2\left(\mathrm{~d}, J_{C P}=14.1 \mathrm{~Hz}\right), 128.7(2 \mathrm{~s}), 129.1,130.7,132.5\left(\mathrm{~d}, J_{C P}=11.8 \mathrm{~Hz}\right), 133.3,134.0,134.3$, $136.0\left(\mathrm{~d}, J_{C P}=11.8 \mathrm{~Hz}\right), 145.9\left(\mathrm{dd}, J_{C P}=20.4,115.6 \mathrm{~Hz}\right), 159.0 ;\left\{{ }^{1} \mathrm{H}\right\}^{31} \mathrm{P} \mathrm{NMR}\left(202.5 \mathrm{MHz}, \mathrm{CD}_{2} \mathrm{Cl}_{2}\right): \delta+26.8$ (s). HRMS calcd for $\mathrm{C}_{39} \mathrm{H}_{39} \mathrm{~N}_{3} \mathrm{O}_{2} \mathrm{P}_{2} \mathrm{~S}_{2} \mathrm{Na}[\mathrm{M}+\mathrm{Na}]^{+} \mathrm{m} / \mathrm{z} 730.18511$, found $\mathrm{m} / \mathrm{z} 730.18415$. Anal. Calcd for $\mathrm{C}_{39} \mathrm{H}_{39} \mathrm{~N}_{3} \mathrm{O}_{2} \mathrm{P}_{2} \mathrm{~S}_{2}$ : C, 66.18; H, 5.55; found C, 66.25; $\mathrm{H}, 5.82$.

$(R, R)$-(2-benzyl)-4,5-bis-[(o-biphenyl)-phenyl-thiophosphinyl]-2H-1,2,3-triazole (13c): Reaction was performed using $0.183 \mathrm{~g}$ of bis(thiophosphinyl)acetylene 12c. Purification was accomplished by column chromatography on silica gel using petroleum ether/dichloromethane (1:3) as eluent. White solid $(0.19 \mathrm{~g}, 86 \%)$. $R_{f} 0.55$ (petroleum ether/dichloromethane 1:3); $\mathrm{mp} 96-98^{\circ} \mathrm{C}(\mathrm{dec}$ ); Enantiomeric excess: $99 \%$ by HPLC analysis (chiralpak IA, $1.0 \mathrm{~mL} \cdot \mathrm{min}^{-1}$, hexane/2-propanol 93:7, $\mathrm{t}_{\mathrm{R}}(R, R)=22.9 \mathrm{~min}, \mathrm{t}_{\mathrm{R}}(S, S)=25.3$ $\left.\min , \mathrm{t}_{\mathrm{R}}(R, S)=36.5 \mathrm{~min}\right) ;[\alpha]_{\mathrm{D}}-38.7\left(\mathrm{c} 0.3, \mathrm{CHCl}_{3}\right) .{ }^{1} \mathrm{H} \mathrm{NMR}\left(600 \mathrm{MHz}, \mathrm{CD}_{2} \mathrm{Cl}_{2}\right)$ : $\delta 5.18(2 \mathrm{AB}$ syst, $J=14.0 \mathrm{~Hz}, 2 \mathrm{H}), 6.79$ (br. t, $J=7.9$ $\mathrm{Hz}, 4 \mathrm{H}), 6.94-6.97(\mathrm{~m}, 6 \mathrm{H}), 7.16-7.18(\mathrm{~m}, 2 \mathrm{H}), 7.22-7.23(\mathrm{~m}, 2 \mathrm{H}), 7.29-7.32(\mathrm{~m}, 4 \mathrm{H}), 7.36-7.45(\mathrm{~m}, 7 \mathrm{H})$, 7.52-7.57 (m, 6H), 7.93-7.97 (m, $2 \mathrm{H}) ;\left\{{ }^{1} \mathrm{H}{ }^{13} \mathrm{C}\right.$ NMR $\left(151 \mathrm{MHz}, \mathrm{CD}_{2} \mathrm{Cl}_{2}\right): \delta 58.7,126.5,126.6,126.8\left(\mathrm{~d}, J_{C P}=14.6 \mathrm{~Hz}\right), 127.4\left(\mathrm{~d}, J_{C P}=13.7 \mathrm{~Hz}\right), 128.6\left(\mathrm{~d}, J_{C P}=3.4 \mathrm{~Hz}\right)$, $128.8,129.8,130.7,131.0,131.3\left(\mathrm{~d}, J_{C P}=90.8 \mathrm{~Hz}\right), 132.0\left(\mathrm{~d}, J_{C P}=11.1 \mathrm{~Hz}\right), 132.6\left(\mathrm{~d}, J_{C P}=11.1 \mathrm{~Hz}\right), 133.7\left(\mathrm{~d}, J_{C P}=28.3 \mathrm{~Hz}\right), 134.2$ (d, $\left.J_{C P}=13.7 \mathrm{~Hz}\right), 134.4,140.5,145.7\left(\mathrm{~d}, J_{C P}=9.9 \mathrm{~Hz}\right), 146.6\left(\mathrm{dd}, J_{C P}=18.5,109.3 \mathrm{~Hz}\right) ;\left\{{ }^{1} \mathrm{H}\right\}^{31} \mathrm{P}$ NMR $\left(243 \mathrm{MHz}, \mathrm{CD}_{2} \mathrm{Cl}_{2}\right): \delta+30.8(\mathrm{~s})$. HRMS calcd for $\mathrm{C}_{45} \mathrm{H}_{35} \mathrm{~N}_{3} \mathrm{P}_{2} \mathrm{~S}_{2} \mathrm{Na}[\mathrm{M}+\mathrm{Na}]^{+} \mathrm{m} / \mathrm{z} 766.16398$, found $\mathrm{m} / \mathrm{z} 766.16352$.

General procedure for the synthesis of P-chirogenic 4,5-bis(phosphino)-1,2,3-triazoles 6: To a solution of 4,5-bis(thiophosphinyl)1,2,3-triazole $13(0.20 \mathrm{mmol})$ in toluene $(4 \mathrm{~mL})$ was added $\mathrm{Si}_{2} \mathrm{Cl}_{6}(0.14 \mathrm{~mL}, 0.80 \mathrm{mmo})$. The resulting mixture was stirred under argon at $80^{\circ} \mathrm{C}$ during one hour. After cooling to $0^{\circ} \mathrm{C}, \mathrm{NaOH}(30 \%$ in water) $(6 \mathrm{~mL})$ was added dropwise and the solution was stirred 5 minutes at 
$0^{\circ} \mathrm{C}$. Water $(10 \mathrm{~mL})$ was added and aqueous phase was extracted with dichloromethane $(3 \times 10 \mathrm{~mL})$. The organic phases were dried over $\mathrm{MgSO}_{4}$, filtered and the solvent evaporated to give a residue which was purified by column chromatography and recrystallization.

(S,S)-(2-benzyl)-4,5-bis-[(2-methoxyphenyl)-phenyl-phosphinyl]-2H-1,2,3-triazole (6a): Reaction was performed using 0.130 g of 4,5-bis(thiophosphinyl)-1,2,3-triazole 13a. Purification was accomplished by column chromatography on silica gel using dichloromethane as eluent and recrystallization in hexane/dichloromethane. White solid $(0.090 \mathrm{~g}, 78 \%)$. $\mathrm{R}_{\mathrm{f}} 0.48$ (dichloromethane); mp $130-132^{\circ} \mathrm{C}$; Enantiomeric excess: $99 \%$ by HPLC analysis (lux $5 \mu$ cellulose-1, $1.0 \mathrm{~mL} \mathrm{~min}^{-1}$, hexane/2-propanol 95:5, $\mathrm{t}_{\mathrm{R}}(R, R)=15.3 \mathrm{~min}, \mathrm{t}_{\mathrm{R}}(S, S)=$ $\left.17.8 \mathrm{~min}, \mathrm{t}_{\mathrm{R}}(R, S)=21.4 \mathrm{~min}\right) ;[\alpha]_{\mathrm{D}}-22.6\left(\mathrm{c} 0.3, \mathrm{CHCl}_{3}\right) .{ }^{1} \mathrm{H} \mathrm{NMR}\left(600 \mathrm{MHz}, \mathrm{CD}_{2} \mathrm{Cl}_{2}\right): \delta 3.53(\mathrm{~s}, 6 \mathrm{H}), 5.67(2 \mathrm{AB} \mathrm{syst}, J=14.9 \mathrm{~Hz}, 2 \mathrm{H})$, 6.78-6.81 (m, 6H), 7.19-7.20 (m, 2H), 7.28-7.41 (m, 15H); $\left\{{ }^{1} \mathrm{H}\right\}^{13} \mathrm{C}$ NMR $\left(125.8 \mathrm{MHz}, \mathrm{CD}_{2} \mathrm{Cl}_{2}\right): \delta 55.2,58.6,110.1,120.6,124.8\left(\mathrm{t}, J_{C P}=\right.$ $3.7 \mathrm{~Hz}), 127.3,128.0,128.1\left(2 \mathrm{~d}, J_{C P}=3.7 \mathrm{~Hz}\right.$ and $\left.J_{C P}=4.1 \mathrm{~Hz}\right), 128.5,128.7,130.0,130.3,134.2\left(\mathrm{t}, J_{C P}=10.9 \mathrm{~Hz}\right), 134.9\left(\mathrm{t}, J_{C P}=3.8\right.$ $\mathrm{Hz}$ ), 135.9, 150.3 (dd, $J_{C P}=5.3,8.7 \mathrm{~Hz}$ ), 160.7 (t, $J_{C P}=7.4 \mathrm{~Hz}$ ); $\left.{ }^{1} \mathrm{H}\right\}^{31} \mathrm{P}$ NMR $\left(243 \mathrm{MHz}, \mathrm{CD}_{2} \mathrm{Cl}_{2}\right): \delta-43.7(\mathrm{~s}) . \mathrm{HRMS}$ calcd for $\mathrm{C}_{33} \mathrm{H}_{32} \mathrm{~N}_{3} \mathrm{O}_{2} \mathrm{P}_{2}[\mathrm{M}+\mathrm{H}]^{+} \mathrm{m} / \mathrm{z}$ 588.19643, found $\mathrm{m} / \mathrm{z}$ 588.19740. Anal. Calcd for $\mathrm{C}_{33} \mathrm{H}_{31} \mathrm{~N}_{3} \mathrm{O}_{2} \mathrm{P}_{2}$ : C, 71.54; $\mathrm{H}, 5.32 ;$ found C, 71.32; $\mathrm{H}, 5.40$.

(S,S)-(2-benzyl)-4,5-bis-[(2-isopropoxyphenyl)-phenyl-phosphinyl]-2H-1,2,3-triazole (6b): Reaction was performed using $0.142 \mathrm{~g}$ of 4,5-bis(thiophosphinyl)-1,2,3-triazole 13b. Purification was accomplished by column chromatography on silica gel using petroleum ether/dichloromethane (1:3) as eluent and recrystallization in hexane/dichloromethane. White solid $(0.090 \mathrm{~g}, 70 \%)$. $\mathrm{R}_{\mathrm{f}} 0.64$ (petroleum ether/dichloromethane 1:3); $\mathrm{mp}<50^{\circ} \mathrm{C}$; Enantiomeric excess: $99 \%$ by HPLC analysis (lux $5 \mu$ cellulose-2, $1.0 \mathrm{~mL} \cdot \mathrm{min}^{-1}$, hexane/2propanol 95:5, $\mathrm{t}_{\mathrm{R}}(S, S)=5.0 \mathrm{~min}, \mathrm{t}_{\mathrm{R}}(R, R)=5.7 \mathrm{~min}, \mathrm{t}_{\mathrm{R}}(R, S)=6.6 \mathrm{~min}$ ); [a $]_{\mathrm{D}}-19.5$ (c $\left.0.3, \mathrm{CHCl}_{3}\right) .{ }^{1} \mathrm{H} \mathrm{NMR}\left(600 \mathrm{MHz}, \mathrm{CD}_{2} \mathrm{Cl}_{2}\right): \delta 0.97(\mathrm{~d}$, $J=6.0 \mathrm{~Hz}, 6 \mathrm{H}$ ), $1.00(\mathrm{~d}, J=6.0 \mathrm{~Hz}, 6 \mathrm{H}), 4.42$ (hept, $J=6.0 \mathrm{~Hz}, 2 \mathrm{H}), 5.65$ (br. s, 2H), 6.68-6.69 (m, 4H), 6.77-6.79 (m, 2H), 7.22-7.36 (m, $13 \mathrm{H}), 7.45-7.46(\mathrm{~m}, 4 \mathrm{H}) ;\left\{{ }^{1} \mathrm{H}\right\}^{13} \mathrm{C}$ NMR $\left(151 \mathrm{MHz}, \mathrm{CD}_{2} \mathrm{Cl}_{2}\right): \delta 21.2,21.5,58.6,70.1,111.9,120.0,126.0\left(\mathrm{t}, J_{C P}=3.2 \mathrm{~Hz}\right), 127.6,127.9(\mathrm{~d}$, $\left.J_{C P}=4.4 \mathrm{~Hz}\right), 128.0\left(\mathrm{~d}, J_{C P}=2.0 \mathrm{~Hz}\right), 128.5,128.7,129.7,133.2,134.7\left(\mathrm{t}, J_{C P}=11.5 \mathrm{~Hz}\right), 135.5\left(\mathrm{t}, J_{C P}=3.2 \mathrm{~Hz}\right), 135.8,150.5\left(\mathrm{~d}, J_{C P}=\right.$ $14.6 \mathrm{~Hz}), 158.9\left(\mathrm{~d}, J_{C P}=7.6 \mathrm{~Hz}\right) ;\left\{{ }^{1} \mathrm{H}\right\}^{31} \mathrm{P}$ NMR $\left(243 \mathrm{MHz}, \mathrm{CD}_{2} \mathrm{Cl}_{2}\right): \delta-43.0$ (s). HRMS calcd for $\mathrm{C}_{39} \mathrm{H}_{40} \mathrm{~N}_{3} \mathrm{O}_{2} \mathrm{P}_{2}[\mathrm{M}+\mathrm{H}]^{+} \mathrm{m} / \mathrm{z} 644.25903$, found $m / z 644.25925$.

(S,S)-(2-benzyl)-4,5-bis-[(o-biphenyl)-phenyl-phosphinyl]-2H-1,2,3-triazole (6c): Reaction was performed using $0.149 \mathrm{~g}$ of 4,5 bis(thiophosphinyl)-1,2,3-triazole 13c. Purification was accomplished by column chromatography on silica gel using petroleum ether/dichloromethane (1:2) as eluent and recrystallization in hexane/dichloromethane. White solid $\left(0.090 \mathrm{~g}, 68 \%\right.$ ). $R_{\mathrm{f}} 0.57$ (petroleum ether/dichloromethane 1:2); mp $64-66^{\circ} \mathrm{C}$ (dec.); Enantiomeric excess: $99 \%$ by HPLC analysis (chiralpak IA, $0.3 \mathrm{~mL} \cdot \mathrm{min}^{-1}$, hexane/2propanol 99:1, $\left.\mathrm{t}_{\mathrm{R}}(R, R)=30.3 \mathrm{~min}, \mathrm{t}_{\mathrm{R}}(S, S)=32.2 \mathrm{~min}, \mathrm{t}_{\mathrm{R}}(R, S)=37.3 \mathrm{~min}\right) ;[\alpha]_{\mathrm{D}}+63.8\left(\mathrm{c} 0.3, \mathrm{CHCl}_{3}\right) .{ }^{1} \mathrm{H} \mathrm{NMR}\left(600 \mathrm{MHz}, \mathrm{CD}_{2} \mathrm{Cl}_{2}\right): \delta$ $5.65(2 \mathrm{AB}$ syst, $J=14.6 \mathrm{~Hz}, 2 \mathrm{H}), 7.07-7.09(\mathrm{~m}, 8 \mathrm{H}), 7.13-7.30(\mathrm{~m}, 2 \mathrm{H}), 7.33-7.36(\mathrm{~m}, 2 \mathrm{H}), 7.39-7.43(\mathrm{~m}, 3 \mathrm{H}) ;\left\{^{1} \mathrm{H}\right\}^{13} \mathrm{C} \mathrm{NMR}(151 \mathrm{MHz}$, $\left.\mathrm{CD}_{2} \mathrm{Cl}_{2}\right): \delta 58.7,127.0\left(\mathrm{~d}, J_{C P}=12.3 \mathrm{~Hz}\right), 127.5,127.7,128.0\left(\mathrm{t}, J_{C P}=3.3 \mathrm{~Hz}\right), 128.1,128.3,128.5,128.6,129.4$ (br. s), 129.8 (br. s), $133.7\left(2 \mathrm{~d}, J_{C P}=10.1 \mathrm{~Hz}\right.$ and $\left.J_{C P}=11.2 \mathrm{~Hz}\right), 134.2,134.3\left(\mathrm{~d}, J_{C P}=4.5 \mathrm{~Hz}\right), 135.7,136.2\left(\mathrm{t}, J_{C P}=3.4 \mathrm{~Hz}\right), 141.7\left(\mathrm{t}, J_{C P}=3.0 \mathrm{~Hz}\right), 147.9$ $\left(\mathrm{t}, J_{C P}=14.5 \mathrm{~Hz}\right), 150.8\left(\mathrm{~d}, J_{C P}=13.1 \mathrm{~Hz}\right) ;\left\{{ }^{1} \mathrm{H}\right\}^{31} \mathrm{P}$ NMR $\left(243 \mathrm{MHz}, \mathrm{CD}_{2} \mathrm{Cl}_{2}\right): \delta-40.9(\mathrm{~s})$. HRMS calcd for $\mathrm{C}_{45} \mathrm{H}_{36} \mathrm{~N}_{3} \mathrm{P}_{2}[\mathrm{M}+\mathrm{H}]^{+} \mathrm{m} / \mathrm{z}$ 680.23790 , found $\mathrm{m} / \mathrm{z} 680.23862$.

Diphosphine palladium complex (14): To a solution of diphosphinotriazole $6 a(0.019 \mathrm{~g}, 0.032 \mathrm{mmol})$ and $\left[\mathrm{Pd}\left(\eta^{3}-\mathrm{C}_{3} \mathrm{H}_{5}\right) \mathrm{Cl}\right]_{2}(0.006 \mathrm{~g}$, $0.016 \mathrm{mmol})$ in methylene chloride $(1 \mathrm{~mL})$ was added a solution of $\mathrm{AgPF}_{6}(0.008 \mathrm{~g}, 0.032 \mathrm{mmol})$ in methanol $(0.3 \mathrm{~mL})$. The mixture was stirred at room temperature in the dark during one hour and the finely divided precipitate was filtered off using a Millipore $4 \mu \mathrm{m}$ filter. Slow addition of diethyl ether to the filtrate induced the formation of a powder which was filtered and washed with diethyl ether. Crystallization in $\mathrm{CH}_{2} \mathrm{Cl}_{2} / \mathrm{Et}_{2} \mathrm{O}$ gave the palladium complex 14. White crystalline solid $(0.022 \mathrm{~g}, 78 \%)$. Mp $198-200^{\circ} \mathrm{C}$ (dec). ${ }^{1} \mathrm{H} \mathrm{NMR}(400$ $\left.\mathrm{MHz}, \mathrm{CD}_{2} \mathrm{Cl}_{2}\right)$ : $\delta 3.06(\mathrm{~s}, 6 \mathrm{H}), 4.14$ (br.s, 4H), 5.68-5.80 (m, 3H), 6.81-6.88 (m, 2H), 6.93-7.05 (m, 4H), 7.23-7.29 (m, 2H), 7.29-7.38 (m, $3 \mathrm{H}), 7.45-7.53(\mathrm{~m}, 2 \mathrm{H}), 7.54-7.68(\mathrm{~m}, 6 \mathrm{H}), 7.84(\mathrm{q}, J=6.9 \mathrm{~Hz}, 4 \mathrm{H}) ;\left\{{ }^{1} \mathrm{H}\right\}^{13} \mathrm{C} N M R\left(100.6 \mathrm{MHz}, \mathrm{CD}_{2} \mathrm{Cl}_{2}\right): \delta 56.0,61.7,72.2\left(\mathrm{t}, J_{C P}=16.6\right.$ $\mathrm{Hz}), 112.4,118.0\left(\mathrm{~d}, J_{C P}=51.4 \mathrm{~Hz}\right), 122.2\left(\mathrm{t}, J_{C P}=4.9 \mathrm{~Hz}\right), 123.0\left(\mathrm{t}, J_{C P}=6.2 \mathrm{~Hz}\right), 127.7\left(\mathrm{~d}, J_{C P}=52.1 \mathrm{~Hz}\right), 128.7,129.6\left(\mathrm{~d}, J_{C P}=2.2\right.$ $\mathrm{Hz}), 130.3\left(\mathrm{t}, J_{C P}=5.8 \mathrm{~Hz}\right), 133.1,133.2\left(\mathrm{t}, J_{C P}=2.7 \mathrm{~Hz}\right), 134.8,135.0,135.2\left(\mathrm{t}, J_{C P}=7.1 \mathrm{~Hz}\right), 154.4\left(\mathrm{~d}, J_{C P}=57.9 \mathrm{~Hz}\right), 161.0$, one $\mathrm{C}$ is not seen; $\left\{{ }^{1} \mathrm{H}\right\}^{31} \mathrm{P}$ NMR $\left(243 \mathrm{MHz}, \mathrm{CD}_{2} \mathrm{Cl}_{2}\right.$ ): $\delta 7.2$ (s), -144.5 (hept, $J=707.4 \mathrm{~Hz}$ ). HRMS calcd for $\mathrm{C}_{38} \mathrm{H}_{36} \mathrm{~N}_{3} \mathrm{O}_{2} \mathrm{P}_{2} \mathrm{Pd}[\mathrm{M}-\mathrm{PF}]^{+} \mathrm{m} / \mathrm{z}$ 734.13121, found $m / z 734.12934$.

Procedure for asymmetric allylic substitution with dimethyl malonate as nucleophile: To a Schlenk tube containing P-chirogenic 4,5-bis(phosphino)-1,2,3-triazoles 6a-c $(0.02 \mathrm{mmol}),\left[\mathrm{Pd}\left(\mathrm{C}_{3} \mathrm{H}_{5}\right) \mathrm{Cl}_{2}\right](0.01 \mathrm{mmol})$ and $(E)$-1,3-diphenylprop-2-en-1-yl acetate $15(0.5$ $\mathrm{mmol}$ ) was added $\mathrm{CH}_{2} \mathrm{Cl}_{2}(4 \mathrm{~mL})$. The mixture was stirred $30 \mathrm{~min}$ at room temperature. Dimethylmalonate $(0.12 \mathrm{~mL}, 1.0 \mathrm{mmol})$ followed by $\mathrm{N}, \mathrm{O}$-bis(trimethylsilyl)acetamide $(0.24 \mathrm{~mL}, 1.0 \mathrm{mmol})$ and a pitch of potassium acetate, were added. After $2 \mathrm{~h}$, the reaction was diluted with diethyl ether and washed with saturated $\mathrm{NH}_{4} \mathrm{Cl}$ solution. The organic phase was dried over $\mathrm{MgSO}_{4}$, filtered and evaporated to give a residue which was purified by chromatography on silica gel using a mixture petroleum ether/ethyl acetate (5:1) as eluent. (E)-Methyl-2-methoxycarbonyl-3,5-diphenylpent-4-enoate 16: ${ }^{1} \mathrm{H} \mathrm{NMR}\left(300 \mathrm{MHz}, \mathrm{CDCl}_{3}\right): \delta 3.56(\mathrm{~s}, 3 \mathrm{H}), 3.75(\mathrm{~s}, 3 \mathrm{H}), 4.02(\mathrm{~d}, J=10.9$ $\mathrm{Hz}, 1 \mathrm{H}), 4.27(\mathrm{dd}, J=8.8,10.8 \mathrm{~Hz}, 1 \mathrm{H}), 6.40(\mathrm{dd}, J=8.6,15.7 \mathrm{~Hz}, 1 \mathrm{H}), 6.54(\mathrm{~d}, J=15.7 \mathrm{~Hz}, 1 \mathrm{H}), 7.10-7.40(\mathrm{~m}, 10 \mathrm{H})$. The enantiomeric excess was determined by HPLC analysis on Chiralpak IA, $1.0 \mathrm{~mL} \cdot \mathrm{min}^{-1}$, hexane/2-propanol 90:10, $\mathrm{t}_{\mathrm{R}}(R)=8.5 \mathrm{~min}, \mathrm{t}_{\mathrm{R}}(S)=10.4 \mathrm{~min}$

Procedure for asymmetric allylic substitution with benzylamine as nucleophile: To a Schlenk tube containing P-chirogenic 4,5bis(phosphino)-1,2,3-triazoles 6a-c $(0.02 \mathrm{mmol}),\left[\mathrm{Pd}\left(\mathrm{C}_{3} \mathrm{H}_{5}\right) \mathrm{Cl}_{2}\right](0.01 \mathrm{mmol})$ and $(E)$-1,3-diphenylprop-2-en-1-yl acetate $15(0.5 \mathrm{mmol})$ was added dichloromethane $(4 \mathrm{~mL})$. The mixture was stirred $30 \mathrm{~min}$ at room temperature and benzylamine $(0.11 \mathrm{~mL}, 1.0 \mathrm{mmol})$ followed by TBAF ( $1 \mathrm{M}$ in THF) $(1.0 \mathrm{~mL}, 1.0 \mathrm{mmol})$ were added. After $18 \mathrm{~h}$, the reaction was diluted with diethyl ether and washed with saturated $\mathrm{NH}_{4} \mathrm{Cl}$ solution. The organic phase was dried over $\mathrm{MgSO}_{4}$, filtered and evaporated to give a residue which was purified by chromatography on silica gel using a mixture of petroleum ether/ethyl acetate $(5: 1)$ as eluent.

(E)-N-Benzyl-(1,3-diphenyl-2-propenyl)amine 17: ${ }^{1} \mathrm{H} \mathrm{NMR}\left(300 \mathrm{MHz}, \mathrm{CDCl}_{3}\right): \delta 1.70(\mathrm{~s}, 1 \mathrm{H}), 3.77(\mathrm{~d}, J=13.2 \mathrm{~Hz}, 1 \mathrm{H}), 3.79(\mathrm{~d}, J=13.2$ $\mathrm{Hz}, 1 \mathrm{H}), 4.40(\mathrm{~d}, J=7.6 \mathrm{~Hz}, 1 \mathrm{H}), 6.32(\mathrm{dd}, J=7.6,15.8 \mathrm{~Hz}, 1 \mathrm{H}), 6.58(\mathrm{~d}, J=15.8 \mathrm{~Hz}, 1 \mathrm{H}), 7.13-7.52(\mathrm{~m}, 15 \mathrm{H})$. The enantiomeric excess was determined by HPLC analysis on Lux $5 \mu$ cellulose-1, $0.5 \mathrm{~mL} \cdot \mathrm{min}^{-1}$, hexane/2-propanol 98:2, $\mathrm{t}_{\mathrm{R}}(R)=20.9 \mathrm{~min}, \mathrm{t}_{\mathrm{R}}(S)=22.2 \mathrm{~min}$.

\section{Acknowledgements}


The authors are grateful for the financial support provided by the CNRS, Ministère de l'Education Nationale et de la Recherche, Université de Bourgogne, Conseil Régional de Bourgogne through the Plan d'Actions Régional pour I'Innovation (PARI) and the Fonds Européen de Développement Régional (FEDER) programs. It is also a pleasure to thank M. J. Penouilh and M. Picquet at the Sayens/Pôle Chimie Moléculaire for the NMR and mass spectrometry analyses, and M. J. Eymin for her skilled technical assistance.

Keywords: P-chirogenic diphosphine $\bullet P, P$-ligand $•$ Palladium • Asymmetric allylic substitution •

[1] a) S. Trofimenko, A. L. Rheingold, C. D. Incarvito, Angew. Chem. Int. Ed. 2003, 42, 3506-3509; Angew. Chem. 2003, 115, 3630-3633. b) E. M. Schuster, M. Botoshansky, M. Gandelman Organometallics 2009, 28, 7001-7005. c) E. M. Schuster, G. Nisnevich, M. Botoshansky, M. Gandelman, Organometallics 2009, 28, 5025-5031. d) N. V. Dubrovina, L. Domke, I. A. Shuklov, A. Spannenberg, R. Franke, A. Villinger, A. Börner, Tetrahedron 2013, 69, 8809-8817. e) D. Mondal, M. S. Balakrishna, Eur. J. Inorg. Chem. 2020, 2392-2402.

[2] E. M. Schuster, M. Botoshansky, M. Gandelman, Angew. Chem. Int. Ed. 2008, 47, 4555-4558.

[3] E. A. Slutsky Smith, G. Molev, M. Botoshansky, M. Gandelman, Chem. Commun. 2011, 47, 319-321.

[4] C. Laborde, M.-M. Wei, A. van der Lee, E. Deydier, J.-C. Daran, J.-N. Volle, R. Poli, J.-L. Pirat, E. Manoury, D. Virieux, Dalton Trans. 2015, 44, 12539-12545.

[5] a) L. Radhakrishna, M. K. Pandey, M. S. Balakrishna, RSC. Adv. 2018, 8, 25704-25718. b) L. Radhakrishna, H. S. Kunchur, P. K. Namdeo, R. J. Butcher, M. S. Balakrishna, Dalton Trans. 2020, 49, 3434-3449..

[6] a) S.-i. Fukuzawa, H. Oki, M. Osaka, J. Sugazawa, S. Kikuchi, Org. Lett. 2007, 9, 5557-5560. b) S.-i. Fukuzawa, H. Oki, Org. Lett. 2008, 10, 1747-1750. c) H. Oki, I. Oura, T. Nakamura, K. Ogata, S.-i. Fukuzawa, Tetrahedron: Asymmetry 2009, 20, 2185-2191.

[7] The same group also reported the use of ClickFerrophos II ligands in asymmetric catalysis. See: T. Konno, K. Shimizu, K. Ogata, S.-i. Fukuzawa, J. Org. Chem. 2012, 77, 3318-3324.

[8] For examples of P-chirogenic 1,2,3-triazole based monophosphines, see : a) F. Dolhem, M. J. Johansson, T. Antonsson, N. Kann, J. Comb. Chem. 2007, 9, 477-486. b) R. Veillard, E. Bernoud, I. Abdellah, J.-F. Lohier, C. Alayrac, A.-C. Gaumont, Org. Biomol. Chem. 2014, 12, 3635-3640. c) J. Bayardon, B. Rousselle, Y. Rousselin, Q. Bonnin, R. Malacea-Kabbara, Eur. J. Org. Chem. 2020, 4723-4729.

[9] For some examples of asymmetric catalysis using P-chirogenic diphosphines, see: a) W.-J Tang, X.-M. Zhang, Chem. Rev. 2003, 103, 3029-3069. b) K. V. L. Crépy, T. Imamoto, Adv. Synth. Catal. 2003, 345, 79-101. c) A. Börner, Phosphorus Ligands in Asymmetric Catalysis, Wiley-VCH, Weinheim, Germany, 2008. d) A. Grabulosa, P-Stereogenic Ligands in Enantioselective Catalysis, RSC, Cambridge, U.K, 2011. e) M. Dutartre, J. Bayardon, S. Jugé, Chem. Soc. Rev. 2016, 45, 5771-5794. f) T. Imamoto, Chem. Rec. 2016, 16, $2659-2673$. g) G. Xu, C. H. Senanayake, W. Tang, Acc. Chem. Res. 2019, 52, 1101-1112.

[10] a) W. S. Knowles, M. J. Sabacky, B. D. Vineyard, D. J. Weinkauff, J. Am. Chem. Soc. 1975, 97, 2567-2568. b) B. D. Vineyard, W. S. Knowles, M. J. Sabacky, G. L. Bachman, D. J. Weinkauff, J. Am. Chem. Soc. 1977, 99, 5946-5952.

[11] For examples of QuinoxP* in asymmetric catalysis, see: a) T. Imamoto, K. Sugita, K. Yoshida, J. Am. Chem. Soc. 2005, 127, 11934-11935. b) T. Imamoto, M. Nishimura, A. Koide, K. Yoshida, J. Org. Chem. 2007, 72, 7413-7416. c) A. Yanagisawa, S. Takeshita, Y. Izumi, K. Yoshida, J. Am. Chem. Soc. 2010, 132, 5328-5329. d) T. Sawano, K. Ou, T. Nishimura, T. Hayashi, Chem. Commun. 2012, 48, 6106-6108. e) H. Le, R. E. Kyne, L. A. Brozek, J. P. Morken, Org. Lett. 2013, 15, 1432-1435. f) X. Wang, S. L. Buchwald, J. Org. Chem. 2013, 78, 3429-3433.

[12] a) Y. Yamanoi, T. Imamoto, J. Org. Chem. 1999, 64, 2988-2989. b) I. D. Gridnev, Y. Yamanoi, N. Higashi, H. Tsuruta, M. Yasutake, T. Imamoto, Adv. Synth. Catal. 2001, 343, 118-136. c) T. Ogura, K. Yoshida, A. Yanagisawa, T. Imamoto, Org. Lett. 2009, 11, 2245-2248.

[13] W. Tang, B. Qu, A. G. Capacci, S. Rodriguez, X. Wei, N. Haddad, B. Narayanan, S. Ma, N. Grinberg, N. K. Yee, D. Krishnamurthy, C. H. Senanayake, Org. Lett. 2010, 12, 176-179.

[14] a) F. Chaux, S. Frynas, H. Laureano, C. Salomon, G. Morata, M.-L. Auclair, M. Stephan, R. Merdes, P. Richard, M.-J. Ondel-Eymin, J.-C. Henry, J. Bayardon, C. Darcel, S. Jugé, C. R. Chimie 2010, 13, 1213-1226. b) N. Khiri-Meribout, E. Bertrand, J. Bayardon, M.-J. Eymin, Y. Rousselin, H. Cattey, D. Fortin, P. D. Harvey, S. Jugé, Organometallics 2013, 32, 2827-2839. c) J. Bayardon, Y. Rousselin, S. Jugé, Org. Lett. 2016, 18, 2930-2933.

[15] E. B. Kaloun, R. Merdès, J.-P. Genêt, J. Uziel, S. Jugé, J. Organomet. Chem. 1997, 529, 455-463.

[16] C. Bauduin, D. Moulin, E. B. Kaloun, C. Darcel, S. Jugé, J. Org. Chem. 2003, 68, 4293-4301.

[17] 2-(trimethylsilyl)ethynyllithium was prepared by a modified literature procedure: D. Prenzel, T. Sander, J. Gebhardt, H. Soni, F. Hampel, A. Görling, S. Maier, R. R. Tykwinski, Chem. Eur. J. 2017, 23, 1846-1852.

[18] An example of P-chirogenic Bis(t-butylmethylphosphino)acetylene and its diborane analogue has been previously reported in literature. See: T. Imamoto, Y. Horiuchi, E. Hamanishi, S. Takeshita, K. Tamura, M. Sugiya, K. Yoshida, Tetrahedron 2015, 71, 6471-6480.

[19] Partial decomplexation of phosphine borane bearing ortho-substituted aryl substituent on P-centre has been already observed in our group. See J. Bayardon, H. Laureano, V. Diemer, M. Dutartre, U. Das, Y. Rousselin, J.-C. Henry, F. Colobert, F. R. Leroux, S. Jugé, J. Org. Chem. 2012, 77, 5759-5769.

[20] A. L. Rheingold, L. M. Liable-Sands, S. Trofimenko, Inorg. Chim. Acta 2002, 330, 38-43.

[21] Compounds 13a-c were obtained as single regioisomer. Only the benzylated N2-position of the triazole ring was detected by ${ }^{1} \mathrm{H}$ NMR in the crude mixture.

[22] G. Zon, K. E. DeBruin, K. Naumann, K. Mislow, J. Am. Chem. Soc. 1969, 91, 7023-7027.

[23] The $(S p, S p)$ configuration of diphosphinotriazole 9a as well as the configuration of the P-center of each intermediate 11a, 13a, 14a, 15a and 16a are in good agreement with the stereochemistry previously reported for the ephedrine methodology (see for example ref [12]) and for the desulfidation with $\mathrm{Si}_{2} \mathrm{Cl}_{6}$ (see ref [14]).

[24] For examples of review on Pd-catalyzed asymmetric allylic substitution reactions, see: a) B. M. Trost, J. E. Schultz, Synthesis 2019, 51, 130. b) B. M. Trost, Org. Process Dev. Res. 2012, 16, 185-194. c) I. Guerrero-Rios, A. Rosas-Hernandez, E. Martin, Molecules 2011, 16, 970-1010. d) B. M. Trost, T. Zhang, J. D. Sieger, Chem. Sci. 2010, 1, 427-440. e) B. M. Trost, M. L. Crawley, Chem. Rev. 2003, 103, 29212943.

[25] For examples of P-chirogenic phosphines in Pd-catalyzed asymmetric allylic asubstitution, see: a) A. Jaillet, C. Darcel, J. Bayardon, A. Schlachter, C. Salomon, Y. Rousselin, P. D. Harvey, S. Jugé, J. Org. Chem. https://dx.doi.org/10.1021/acs.joc.0c00536. b) J. Bayardon, M. Maronnat, A. Langlois, Y. Rousselin, P. D. Harvey, S. Jugé, Organometallics, 2015, 34, 4340-4358. c) X.-F. Yhang, W.-H. Yu, C.-H. Ding, 
Q.-P. Ding, S.-L. Wan, X.-L. Hou, L.-X. Dai, P.-J. Wang, J. Org. Chem. 2013, 78, 6503-6509. d) K. Zhang, Q. Peng, X.-L. Hou, Y.-D. Wu, Angew. Chem. Int. Ed. 2008, 47, 1741-1744. e) T. Imamoto, M. Nishimura, A. Koide, K. Yoshida, J. Org. Chem. 2007, 72, 7413-7416. f) N. Oohara, K. Katagiri, T. Imamoto, Tetrahedron: Asymmetry, 2003, 14, 2171-2175. g) U. Nettekoven, M. Widhalm, H. Kalchhauser, P. C. J. Kamer, P. W. N. M. van Leeuven, M. Lutz, A. L. Spek, J. Org. Chem. 2001, 66, 759-770. h) H. Tsuruta, T. Imamoto, Synlett 2001, 9991002.

[26] D. A. Evans, K. R. Campos, J. S. Tedrow, F. E. Michael, M. R. Gagné, J. Am. Chem. Soc. 2000, 122, 7905-7920.

[27] J. Holz, K. Rumpel, A. Spannenberg, R. Paciello, H. Jiao, A. Börner, ACS Catal. 2017, 7, 6162-6169.

[28] E. A. Colby, T. F. Jamison, J. Org. Chem. 2003, 68, 156-166. 